\title{
Applications of Bayesian Shrinkage Prior Models in Clinical Research with Categorical Responses
}

\author{
Arinjita Bhattacharyya \\ University of Louisville \\ Subhadip Pal \\ University of Louisville \\ Riten Mitra \\ University of Louisville \\ Shesh Rai ( $\nabla$ shesh.rai@louisville.edu ) \\ University of Louisville
}

\section{Research Article}

Keywords: shrinkage priors, logistic regression, horseshoe, Dirichlet Laplace, MCMC, Polya-Gamma, multinomial, ADNI, Pima, data augmentation

Posted Date: January 5th, 2021

DOI: https://doi.org/10.21203/rs.3.rs-137866/v1

License: (1) (1) This work is licensed under a Creative Commons Attribution 4.0 International License. Read Full License 


\title{
RESEARCH
}

\section{Applications of Bayesian Shrinkage prior models in clinical research with categorical responses}

\author{
Arinjita. Bhattacharyya ${ }^{1}$, Subhadip Pal ${ }^{1 \dagger}$, Riten Mitra ${ }^{1 \dagger}$ and Shesh N. Rai ${ }^{1,2,3,4,5^{*}}$
}

\footnotetext{
${ }^{*}$ Correspondence:

shesh.rai@louisville.edu

${ }^{1}$ Department of Bioinformatics \&

Biostatistics, University of

Louisville, KY, USA

Full list of author information is

available at the end of the article

${ }^{\dagger}$ Equal contributor
}

\begin{abstract}
Background: Prediction and classification algorithms are commonly used in clinical research for identifying patients susceptible to clinical conditions like diabetes, colon cancer, and Alzheimer's disease. Developing accurate prediction and classification methods have implications for personalized medicine. Building an excellent predictive model involves selecting features that are most significantly associated with the response at hand. These features can include several biological and demographic characteristics, such as genomic biomarkers and health history. Such variable selection becomes challenging when the number of potential predictors is large. Bayesian shrinkage models have emerged as popular and flexible methods of variable selection in regression settings. The article discusses variable selection with three shrinkage priors and illustrates its application to clinical data sets such as Pima Indians Diabetes, Colon cancer, ADNI, and OASIS Alzheimer's data sets.
\end{abstract}

Methods: We present a unified Bayesian hierarchical framework that implements and compares shrinkage priors in binary and multinomial logistic regression models. The key feature is the representation of the likelihood by a Polya-Gamma data augmentation, which admits a natural integration with a family of shrinkage priors. We specifically focus on the Horseshoe, Dirichlet Laplace, and Double Pareto priors. Extensive simulation studies are conducted to assess the performances under different data dimensions and parameter settings. Measures of accuracy, AUC, brier score, L1 error, cross-entropy, ROC surface plots are used as evaluation criteria comparing the priors to frequentist methods like Lasso, Elastic-Net, and Ridge regression.

Results: All three priors can be used for robust prediction with significant metrics, irrespective of their categorical response model choices. Simulation study could achieve the mean prediction accuracy of $91 \%(95 \% \mathrm{Cl}: 90.7,91.2)$ and $74 \%(95 \% \mathrm{Cl}: 73.8,74.1)$ for logistic regression and multinomial logistic models, respectively. The model can identify significant variables for disease risk prediction and is computationally efficient.

Conclusions: The models are robust enough to conduct both variable selection and future prediction because of their high shrinkage property and applicability to a broad range of classification problems.

Keywords: shrinkage priors; logistic regression; horseshoe; Dirichlet Laplace; MCMC; Polya-Gamma; multinomial; ADNI; Pima; data augmentation

\section{Introduction}

Recent innovations and availability of structured electronic health records (EHRs), and multi-site longitudinal studies, high-throughput sequencing (HTS) [1] data have 
made patient characteristics, genomics information accessible for statistical prediction analysis and clinical research. Medical decisions are modified upon the identification of essential covariates for a specific clinical outcome. Treatment recommendations are also age, population, and comorbidity specific, with HTS bringing a paradigm shift in large-scale precision/personalized medicines. For example, in diabetes prediction, various biological and clinical factors like sex, age, obesity are responsible for predicting the future risk of the disease. In oncology, for instance, in colorectal cancer [2], breast cancer [3], non-small cell lung cancer(NSCLS) [4] prognostic, and predictive genes like human epidermal growth factor receptor 2 (HER2), BRAF, KRAS are not only essential for early detection but also therapy selection, subgroup stratification, and controlled monitoring of the disease. In Alzheimer's diseases $(\mathrm{AD})$, a combination of traditional risk factors including age, education, hypertension, obesity, cognitive test scores, cardiovascular disease [5] along with testing of ApoE4 gene can be informative in identifying if a patient is vulnerable to the non-curable disease or any other forms of dementia.

A clinical prediction or classification model, followed by a variable selection strategy, plays a vital role in designing preventive measures from adverse outcomes. Variable selection methods can mitigate noise imposed by irrelevant variables in the data. Most of these methods are frequentist approaches like Lasso [6], ElasticNet[7] that uses an L1 or L2 penalty to shrink the coefficients of irrelevant variables. Bayesian regression methods are a reasonable alternative approach that can incorporate shrinkage properties as prior information is the ultimate focus of this research work.

Public health researchers often face prediction problems with categorical responses depended on the clinical features the primary outcome needs to be predicted, such as the classification of a Covid-19 patient as "discharged" or "died" or "remained in hospital" with individual-patient level data [8]. When the response variable is binary, logistic regression (LR) models enable assessing the association between an independent variable(s) and the response variable and prediction. When the response variable has more than two categories, generalizations of the logistic model, such as multinomial logistic regression (MLR) models, are common. The previous scenarios provide a better understanding of categorical response models and their contribution as a natural and attractive approach in a diverse array of applications, ranging from spam detection, credit card fraud to predicting Alzheimer's stage and tumor malignancy. The categorical response models' primary goal is to infer relationships between a categorical response and a set of explanatory variables. Like any form of regression technique, this is critical to understanding the causal dynamics of a complex system and making accurate predictions on future data. Depending on a specific problem, the explanatory variables or predictors can take on the form of demographic profiles, socio-economic data, or complex bio-molecular entities. Modeling becomes particularly challenging when the number of such predictors grows large, a common feature in genomics. Traditionally, frequentist likelihood-based methods have been using penalized regression techniques to address this "curse of dimensionality" to deal with data having a considerable number of predictors in linear and multi-category response regression. These methods briefly add penalty terms to the negative log-likelihood function and then optimize this penalized likelihood for parameter inference. The penalization leads to a desired "shrinking" 
behavior of the estimates. Specifically, it pulls the small coefficients towards zero while minimally affecting the large coefficients, thus selecting only the most relevant variables. For a LR set up, the objective function that needs to be minimized is of the form

$$
l_{\lambda}^{L R}(\beta)=\sum_{i=1}^{n} y_{i} x_{i} \beta-\log \left(1+e^{x_{i}^{T} \beta}\right)+\lambda\left(\sum_{j=1}^{p}\left|\beta_{j}\right|^{r}\right)^{\frac{1}{r}}, r>0 .
$$

Here, $y=\left(y_{1}, y_{2}, \ldots, y_{n}\right)$ is an $n$ - dimensional vector representing the outcome variable; $x_{i}=\left(x_{i 1}, x_{i 2}, \ldots, x_{i p}\right)$ are the covariates with $x_{i j}$ as the observed values on the $p$ predictors and $\left(\beta_{1}, \beta_{2}, \ldots, \beta_{p}\right)^{T}$ is a $p$-dimensional parameter vector of regression coefficients. $\lambda$ is the penalty parameter which modulates the amount of shrinkage. Large values of $\lambda$ lead to more shrinkage, while $\lambda=0$ leads to the simple logistic likelihood without shrinkage. The generalized objective function for multiple categories will be

$$
l_{\lambda}^{M L R}(\beta)=\sum_{i=1}^{N} y_{i}\left(\sum_{k=0}^{K} x_{i k} \beta_{k}-n_{i} \log \left(1+e^{\sum_{k=0}^{K} x_{i k} \beta_{k}}\right)+\lambda\left(\sum_{k=1}^{K} \sum_{j=1}^{p}\left|\beta_{k j}\right|^{r}\right)^{\frac{1}{r}} .\right.
$$

Different values of $r$ lead to various penalization techniques. For example, $r=1$ results in the well-known Least Absolute Shrinkage and Selection Operator (Lasso) [6] solution and $r=2$ results in the Ridge regression solution [9]. Elastic-Net[7] is another method that is a convex combination of Lasso and Ridge. Bayesian variable selection models form a natural counterpart to penalization, as mentioned earlier techniques with some specialized priors assuming the penalty terms' role. Some notable work in this area include $[10,11,12,13,14,15,16,6,17,18]$ among many. A comprehensive overview of shrinkage priors with data applications is given in [19]. The historical development of shrinkage priors can be traced back to the spike and slab approach proposed in [20]. It is a hierarchical mixture model that essentially uses a latent binary indicator to differentiate between the large and small regression coefficients. It does so by assigning Gaussian priors with high and low variances to the coefficients, conditional on these indicators. The resultant marginal prior for a coefficient takes on a shape with a "spike" at 0 and a relatively flat "slab" at non-zero regions, lending the model its name. [21] suggested stochastic search variable selection (SSVS) for identifying a subset of significant covariates with a hierarchical normal mixture model structure, similar to the spike and slab prior. However, it uses continuous Gaussian distribution to include and exclude variables and lower variance, respectively. The spike-and slab prior has a bi-separation effect on the model coefficients, thus bringing a computational complexity of $2^{p}$. SSVS is computationally intensive and cannot handle a huge set of predictors. Inclusion of non-conjugate and conjugate priors are detailed in this extension of the work [22]. A related class of variable selection (VS) models put positive prior mass at 0 . Inference in these models usually relies on Reversible Jump sampling techniques [23]. Though these models have the nice property of explicitly reducing coefficients to zero, they typically incur high computational costs and exhibit poor mixing. These 
issues necessitated using a computationally efficient class of priors, which allowed sufficient shrinkage in high dimensions. The focus solely is on such priors for this article. These priors are called global-local(GL) shrinkage priors [24]. Denoting the parameter set as $\beta=\left(\beta_{1}, \ldots, \beta_{p}\right)^{T}$, the GL framework can be written hierarchically as follows

$$
\begin{gathered}
\beta_{j} \sim N\left(0, \lambda_{j} \tau\right) \\
\lambda_{j} \sim f(.) \text { and } \tau \sim g(.) .
\end{gathered}
$$

The global parameter $\tau$ controls the overall shrinkage of all the coefficients towards zero while the local parameters $\lambda=\left(\lambda_{1}, \ldots, \lambda_{p}\right)$ modulate the coefficient-specific shrinkage. It is desired that the distribution of the local parameter $f($.$) should$ have heavy tails and the distribution of the global parameter $g($.$) have substan-$ tial mass at point zero. The Normal-Gamma prior [25], the Dirichlet Laplace (DL) prior [18], Horseshoe prior [26], and the Double Pareto (DP) prior [17] are some of the GL priors. A review and comparison of shrinkage methods for VS are detailed in [27]. The second piece of our proposed approach focuses on a convenient data-augmentation representation of the logistic regression likelihood. Two data augmentation algorithms that have gained popularity for binary responses are one for the probit regression model that uses truncated normal random variables [28] and another for logistic regression that uses Polya-Gamma (PG) random variables [29]. Here we focus on the latter scheme. This article's outline follows: In Sections , and the PG Data Augmentation is described under LR and MLR models. Next, the data-augmented likelihood framework's connection to the Horseshoe, DL, and DP priors is presented, embedding them in a fully Bayesian hierarchical model. Performance metrics evaluate the simulation results with different sample sizes, covariate dimensions, and parameter settings with data application in section. Data applications are presented in Section. The limitations and contributions are in the Discussion Section and the article is concluded in Section ??.

\section{Bayesian Binary Regression and Polya-Gamma Data Augmentation}

Consider the binary logistic regression model where $Y_{1}, Y_{2}, \ldots, Y_{n}$ are i.i.d. Bernoulli random variables with variables $\left\{x_{i} \in \mathbb{R}^{p}\right\}_{i=1}^{n}$ as the covariates and $\beta \in \mathbb{R}^{p}$ denotes the unknown regression coefficients. The corresponding likelihood is given by

$$
\prod_{i=1}^{n} P\left(Y_{i}=y_{i} \mid \beta\right)=\prod_{i=1}^{n} \frac{\exp \left(x_{i}^{T} \beta y_{i}\right)}{1+\exp \left(x_{i}^{T} \beta\right)}
$$

In the context of the Bayesian analysis, the posterior of $\beta$ given the data is given by $\pi(\beta \mid Y) \propto \pi(\beta) \prod_{i=1}^{n} \frac{\exp \left(x_{i}^{T} \beta y_{i}\right)}{1+\exp \left(x_{i}^{T} \beta\right)}$, where $\pi(\beta)$ denotes the prior distribution for $\beta$. Unfortunately, the above posterior becomes intractable due to the presence of the term $\left(1+\exp \left(x_{i}^{T} \beta\right)\right)$ in its denominator [30]. Therefore, posterior sampling in this setup traditionally relied on the Metropolis Hastings (MH) algorithm. As an alternative, the Data Augmentation(DA) algorithm utilizes the latent variables to circumvent the difficulty. Before introducing to the specific Polya-Gamma DA scheme that we used in this article, we provide the general structure of a DA algorithm. Suppose, we need to sample a parameter of interest $\theta$ from an intractable 
density $\pi(\theta)$. The technique requires to design a suitable joint density $\pi(\theta, W)$ in such a way that it satisfies the following two criteria ; firstly, $\int \pi(\theta, W) d W=\pi(\theta)$ and secondly, the corresponding conditional distributions, $\pi(\theta \mid W)$ and $\pi(W \mid \theta)$ are possible to sample from [31, 32]. In the context of the Bayesian analysis, $\pi(\theta)$ typically refers to a posterior density for a parameter of interest $\theta$. A major challenge for designing a DA is to construct an appropriate choice for $\pi(\theta, W)$ (see [28], [29]). A commonly used strategy is to build a conditional distribution $\pi(W \mid \theta)$ so that $\pi(\theta, W)=\pi(W \mid \theta) \pi(\theta)$ fulfills the requirements. The latent variable $W$ is often termed as the augmented random variable. In the current context, our parameter of interest is $\beta$ with the posterior density $\pi(\beta \mid Y)$ while we make use of the DA technique designed in [29] where the authors develops and utilizes the PolyaGamma (PG) distribution as the choice for the augmented random variable. As we will require in the later sections, we include the the probability density function of the Polya-Gamma distribution (denoted by $P G(1, c)$ ) [29], as following

$$
\begin{aligned}
p(x \mid c) & =\cosh \left(\frac{c}{2}\right) \exp \left(-\frac{c^{2} x}{2}\right) h(x) \text { where } \\
h(w) & =\sum_{k=0}^{\infty}(-1)^{k} \frac{2 k+1}{\sqrt{2 \pi w}} \exp \left(-\frac{(2 k+1)^{2}}{8 w}\right), 0<w<\infty .
\end{aligned}
$$

To deal with the intractability in Equation 3, the DA scheme augments independent latent variables, $\left\{W_{i}\right\}_{i=1}^{n}$ where $W_{i} \sim P G\left(1,\left|x_{i}^{T} \beta\right|\right)$. $\left\{W_{i}\right\}_{i=1}^{n}$ are also assumed to be independent of $\left\{Y_{i}\right\}_{i=1}^{n}$ for $i=1, \ldots n$. Then, the joint posterior $\pi\left(\beta,\left\{W_{i}\right\}_{i=1}^{n} \mid\left\{Y_{i}\right\}_{i=1}^{n}\right)$ satisfies the two criteria related to a generic DA algorithm that are mentioned above. The integrability criterion trivially holds whereas the the random variables $W_{1}, \ldots, W_{n}$ given $\left\{Y_{i}\right\}_{i=1}^{n}, \beta$ are independent and follows PG distribution [29]. The posterior conditional for $\beta \mid\left\{Y_{i}\right\}_{i=1}^{n},\left\{W_{i}\right\}_{i=1}^{n}$ becomes to be the Multivariate Normal if the Multivariate Normal prior is used for $\beta$ [29]. The appearance of Normal distribution as a posterior for $\beta$ is a key feature which provides a way to utilize more nontrivial priors for $\beta$ without much difficulty. specifically, in this manuscript we use the Global-Local priors that we discuss in details in the next section. As mentioned earlier, Bayesian regression for binary responses has been recognized as a hard problem due to the likelihood's unwieldy form. There have been several efforts towards an improved version of the DA algorithm [28] for probit regression. Some notable works include [33] and [34]. However, these algorithms are more imprecise versions of [28] making it significantly difficult with multiple layers of latent variables and restricting its usage. In contrast, the DA algorithm by [29] is free of these problems and computationally much less cumbersome.

\section{Logistic Regression Model with Hierarchical Prior Structures}

In this subsection, we include the details of each of the three priors distributions along with the corresponding posterior distributions. The original form of the horseshoe prior [35] is represented as

$$
\beta_{j} \mid \Lambda_{j}^{2} \tau^{2} \sim N_{p}\left(0, \Lambda_{j}^{2} \tau^{2}\right), j=1,2, \ldots, p ; \Lambda_{j}^{2} \sim C^{+}(0,1) ; \tau \sim C^{+}(0,1)
$$


Another computationally feasible hierarchical representation of the Horseshoe prior [14] that is used here is

$$
\begin{aligned}
\beta_{j} \mid \Lambda_{j}^{2} \tau^{2} \sim & N_{p}\left(0, \Lambda_{j}^{2} \tau^{2}\right), j=1,2, \ldots, p ; \Lambda_{j}^{2} \mid \gamma_{j} \sim I G\left(\frac{1}{2}, \frac{1}{\gamma_{j}}\right) \\
& \tau^{2} \mid \xi \sim I G\left(\frac{1}{2}, \frac{1}{\xi}\right) ; \gamma_{1}, \gamma_{2}, \ldots, \gamma_{p}, \xi \sim I G\left(\frac{1}{2}, 1\right)
\end{aligned}
$$

Here, $\Sigma$ of the distribution of $\beta$ is a diagonal matrix with elements $\left(\Lambda_{1} \tau^{2}, \ldots \Lambda_{p} \tau^{2}\right)$. From equations $(3),(4)$ and the above hierarchical prior structure $(6)$, the full posterior distribution is given by:

$$
\begin{array}{r}
\pi\left(\beta, \Lambda_{j}^{2}, w_{i}, \gamma_{j}, \tau^{2}, \xi \mid Y\right) \propto \prod_{i=1}^{n} \frac{\exp \left(x_{i}^{T} \beta y_{i}\right)}{\left(1+\exp \left(x_{i}^{T} \beta\right)\right)}\left(1+\exp \left(x_{i}^{T} \beta\right)\right) h\left(w_{i}\right) \\
\exp \left(-\frac{\left(x_{i}^{T} \beta\right)^{2} w_{i}}{2}\right) \frac{\exp \left(-\frac{1}{2}\left(\beta^{T} \Sigma^{-1} \beta\right)\right)}{\sqrt{2 \pi \Sigma}} \\
\prod_{j=1}^{p} \frac{\left(\Lambda_{j}^{2}\right)^{-\left(\frac{1}{2}+1\right)} \exp \left(\frac{-1}{\gamma_{j} \Lambda_{j}^{2}}\right)}{\gamma_{j}^{\frac{1}{2}}} \frac{\left(\tau^{2}\right)^{-\left(\frac{1}{2}+1\right)} \exp \left(\frac{-1}{\tau^{2} \xi}\right)}{\xi^{\frac{1}{2}}} \\
\gamma_{j}^{-\left(\frac{1}{2}+1\right)} \exp \left(\frac{-1}{\gamma_{j}}\right) \xi^{-\left(\frac{1}{2}+1\right)} \exp \left(\frac{-1}{\xi}\right) .
\end{array}
$$

where, $h\left(w_{i}\right)$ is defined in equation (4) The conditional distributions required for our analysis follows:

The conditional density of $\beta$ given $y, w$ is

$$
\pi\left(\beta \mid \Sigma, W_{D}, Y\right) \sim N_{p}\left(\left(X^{T} W_{D} X+\Sigma^{-1}\right)^{-1} X^{T} y^{*},\left(X^{T} W_{D} X+\Sigma^{-1}\right)^{-1}\right)
$$

where, $W_{D}$ and $\Sigma$ are diagonal matrices where the elements are $\left(w_{1}, w_{2}, \ldots, w_{n}\right)$, $\left(\Lambda_{1}^{2} \tau^{2}, \ldots, \Lambda_{p}^{2} \tau^{2}\right)$ respectively and, $y^{*}=\left(y_{1}-\frac{1}{2}, \ldots, y_{n}-\frac{1}{2}\right)$.

In high-dimensional scenarios where sampling of $\beta$ can be difficult and tricky for $p>n$ case, fast sampling technique with Gaussian scale-mixture priors [36] is used where the mean and variance of a Gaussian distribution is in the respective form: $N_{p}(\mu, \hat{\Sigma}), \mu=\hat{\Sigma} A^{T} \alpha, \hat{\Sigma}=\left(A^{T} A+D^{-1}\right)^{-1} D \in R^{p \times p}, A \in R^{n \times p}, \alpha \in R^{n \times 1}$ The algorithm involves sampling $u \sim N(0, D)$, and $\delta \sim N\left(0, I_{n}\right)$; with $V=A u+\delta$ getting the inverse of $\left(A D A^{T}+I_{n}\right) w=(\alpha-v)$, and finally obtaining $\theta=u+D A^{T} w, \theta \sim$ $N(\mu, \Sigma)$. The conditional density of $w_{i}$ given $x_{i}, \beta$ is

$$
\pi\left(w_{i} \mid \beta\right) \sim P G\left(1, x_{i}^{T} \beta\right)
$$


The conditional density of the hyper-parameters are as follows

$$
\begin{array}{r}
\pi\left(\Lambda_{j}^{2} \mid \gamma_{j}, \beta_{j}, \xi, \Lambda_{j}^{2}\right) \sim I G\left(1, \frac{1}{\gamma_{j}}+\frac{\beta_{j}^{2}}{2 \tau^{2}}\right) \\
\pi\left(\gamma_{j} \mid \Lambda_{j}^{2}, \beta_{j}, \xi, \tau^{2}\right) \sim I G\left(1,1+\frac{1}{\Lambda_{j}^{2}}\right) \\
\pi\left(\tau^{2} \mid \gamma_{j}, \beta_{j}, \xi, \Lambda_{j}^{2}\right) \sim I G\left(\frac{p+1}{2}, \frac{1}{\xi}+\sum_{j=1}^{p} \frac{\beta_{j}^{2}}{2 \Lambda_{j}^{2}}\right) \\
\pi\left(\xi \mid \tau^{2}\right) \sim I G\left(1,1+\frac{1}{\tau^{2}}\right) .
\end{array}
$$

Here, all the posterior densities are in the closed form, and follow simple densities like Normal, Polya-Gamma and Inverse-Gamma making sampling from them trivial. The hierarchical structure of the Dirichlet Laplace prior [18] is

$$
\beta_{j} \sim N_{p}\left(0, \psi_{j} \phi_{j}^{2} \tau^{2}\right) ; \psi_{j} \sim \exp \left(\frac{1}{2}\right) ; \phi \sim \operatorname{Dir}(a, a, \ldots, a) ; \tau \sim G\left(p a, \frac{1}{2}\right)
$$

The conditional posterior distributions remain same for $\beta \mid y_{i}$ and $w_{i} \mid \beta$ is similar to that of equations (8) and (9).

The conditional density of the hyper-parameters as obtained similar to Theorem 2.2 in [18] are as follows:

$$
\begin{array}{r}
\pi(\psi \mid \phi, \tau, \beta) \sim I G\left(\frac{\phi_{j} \tau}{\left|\beta_{j}\right|}, 1\right) \\
\pi(\tau \mid \phi, \beta) \sim G I G\left(p a-p, 1,2 \sum_{j=1}^{p} \frac{\left|\beta_{j}\right|}{\phi_{j}}\right) .
\end{array}
$$

To sample $\pi\left(\phi \mid \beta_{j}\right)$ sample $T_{j} \sim G I G\left(a-1,1,2\left|\beta_{j}\right|\right)$, set $\phi_{j}=\frac{T_{j}}{T}, T=\sum_{j=1}^{p} T_{j}$. where $\operatorname{GIG}(a, b, c)$ is the Generalized Inverse Gaussian distribution with density $f(x ; a, b, c) \propto x^{(c-1)} e^{\frac{-1}{2}\left(a x+\frac{b}{x}\right)}$.

The hierarchical structure of Double Pareto prior[28] is

$\beta\left|\Lambda, \tau \sim N_{p}\left(0, D_{\tau}\right) ; w_{i}\right| x_{i}, \beta \sim P G\left(1, x_{i}^{T} \beta\right) ; \tau_{j} \mid \Lambda_{j} \sim \exp \left(\frac{\Lambda^{2}}{2}\right) ; \Lambda_{j} \sim G(\zeta, \eta)$

Again, the conditional densities of $\beta \mid y_{i}$ and $w_{i} \mid \beta$ remains same as (8) and (9). Here $\Sigma=D_{\tau}$ is a diagonal matrix with elements $\left(\tau_{1}, \tau_{2}, \ldots, \tau_{p}\right)$. The conditional density of rest of the hyper-parameters are as follows:

$$
\begin{array}{r}
\pi\left(\tau_{j} \mid \beta, \Lambda, y\right) \sim \operatorname{GIG}\left(\frac{1}{2}, \Lambda_{j}^{2}, \beta_{j}^{2}\right) \\
\pi(\Lambda \mid \beta, y) \sim \operatorname{Gamma}\left(\zeta+1, \eta+\left|\beta_{j}\right|\right) .
\end{array}
$$




\section{Bayesian Multinomial Logistic Regression and Polya-Gamma Data Augmentation}

This is the extension of the binary regression, where the outcome variable has more than two classes. Let $Y_{i}, i=1,2, \ldots, n$ be a categorical random variable with $k$ categories, where $k \geq 2$. The probability for the $k$-th category is $p_{i k}=P\left(Y_{i}=k \mid\right.$ $x_{i}$ ), where $\sum_{k=1}^{K} p_{i k}=1$. The multinomial logistic regression (MLR) model is given as

$$
\begin{aligned}
P\left(Y_{i}=k \mid x_{i}\right) & =p_{i k} \quad=\frac{e^{x_{i}^{T} \beta_{k}}}{1+\sum_{j=1}^{K-1} e^{x_{i}^{T} \beta_{j}}}, k=1,2, \ldots K-1 \\
P\left(Y_{i}=K \mid x_{i}\right) & =p_{i K}=\frac{1}{1+\sum_{j=1}^{K-1} e^{x_{i}^{T} \beta_{j}}} .
\end{aligned}
$$

Here, $\beta_{k}$ are the coefficients associated with $k$-th category, and $K$ is the baseline category with its coefficient $\beta_{K}$ constrained to zero. The Polya-Gamma data-augmentation approach can be extended for the purpose of multi-category response variable. Here, $\beta_{k}$ is updated conditional on the remaining $\beta_{-k}=$ $\left(\beta_{1}, \beta_{2}, \ldots, \beta_{k-1}, \beta_{k+1}, \ldots, \beta_{K}\right)$. The full conditional for $\beta_{k}$ given $y$ and $\beta_{j \neq k}$ can be expressed as a likelihood of the Bernoulli distribution.

$$
f\left(\beta_{k} \mid y, \beta_{-k}\right) \propto f\left(\beta_{k}\right) \Pi_{i=1}^{n} p_{i k}^{I\left(y_{i}=k\right)}\left(1-p_{i k}\right)^{1-I\left(y_{i}=k\right)}
$$

where $f\left(\beta_{k}\right)$ is the prior for coefficient $\beta_{k}, I\left(Y_{i}=k\right)=1$ when $Y_{i}=k$ with probability $p_{i k}=P\left(Y_{i}=k\right)=\frac{e^{x_{i}^{T} \beta_{k}}}{\sum_{j=1}^{K} e^{x_{i}^{T} \beta_{k}}}$. It can be re-written as $p_{i k}=P\left(Y_{i}=\right.$ $k)=\frac{e^{x_{i}^{T} \beta_{k}-M_{i k}}}{1+e^{x_{i}^{T} \beta_{k}-M_{i k}}}=\frac{e^{\psi_{i k}}}{1+e^{\psi_{i k}}}$ where, $M_{i k}=\log \sum_{j \neq k} e^{x_{i}^{T} \beta_{j}}$ and $\psi_{i k}=x_{i}^{T} \beta_{k}-M_{i k}$. $\sum_{j \neq k} e^{x_{i}^{T} \beta_{j}}$ includes the reference category $K$, as $\beta_{K}=0$, so $e^{x_{i}^{T} \beta_{k}}=1$, and hence $M_{i k}=\log \sum_{j \neq k} e^{x_{i}^{T} \beta_{j}}=\log \left(1+\sum_{j \neq k, K} e^{x_{i}^{T} \beta_{j}}\right)$. Thus, the full conditional for $\beta_{k}$ given $y$ and $\beta_{-k}$ is

$f\left(\beta_{k} \mid y, \beta_{-k}\right) \propto f\left(\beta_{k}\right) \Pi_{i=1}^{n}\left(\frac{e^{\psi_{i k}}}{1+e^{\psi_{i k}}}\right)^{I\left(y_{i}=k\right)}\left(\frac{1}{1+e^{\psi_{i k}}}\right)^{1-I\left(y_{i}=k\right)}=f\left(\beta_{k}\right) \Pi_{i=1}^{n} \frac{\left(e^{\psi_{i k}}\right)^{I\left(y_{i}=k\right)}}{1+e^{\psi_{i k}}}$.

which is a logistic regression likelihood, giving ways to use PG data-augmentation to update each of $\beta_{k}$ 's based on the binary indicator variable $I\left(y_{i}=k\right)$.

Multinomial Logistic Regression with Hierarchical Prior Structures

The hierarchical structures with the three priors will be calculated similarly as Section . Considering $N\left(0, \Lambda_{t}^{2} \tau^{2}\right), t=1,2, \ldots, p$, for $\beta_{1}, \beta_{2}, \ldots, \beta_{K-1}$, the conditional density for $\beta_{k}$ and $w_{i k}$ are given as

$\beta_{k} \sim N\left(\mu_{k}, \Sigma_{k}\right), \mu_{k}=\Sigma_{k}\left(\Sigma_{0}+X^{T} W_{k} y_{k}^{*}\right), \Sigma_{k}=\left(\Sigma_{0}+X^{T} W_{k} X\right)^{-1} w_{i k} \sim P G\left(1, \psi_{i k}\right)$ 
where $\Sigma_{0}=\operatorname{diag}\left(\Lambda_{t}^{2} \tau^{2}\right), W_{k}=\operatorname{diag}\left(w_{i k}\right), y_{k}^{*}=\frac{I\left(y_{i}=k\right)-0.5}{w_{i k}}+M_{i k}$.

The conditional density of the hyper-parameters in case of Horseshoe prior is

$$
\begin{array}{r}
\pi\left(\Lambda_{t k}^{2} \mid \gamma_{t k}, \beta_{t k}, \xi, \Lambda_{t k}^{2}\right) \sim I G\left(1, \frac{1}{\gamma_{t k}}+\frac{\beta_{t k}^{2}}{2 \tau^{2}}\right) \\
\pi\left(\gamma_{t k} \mid \Lambda_{t k}^{2}, \beta_{t k}, \xi, \tau^{2}\right) \sim I G\left(1,1+\frac{1}{\Lambda_{t k}^{2}}\right) \\
\pi\left(\tau^{2} \mid \gamma_{t k}, \beta_{t k}, \xi, \Lambda_{t k}^{2}\right) \sim I G\left(\frac{p+1}{2}, \frac{1}{\xi}+\sum_{t=1}^{p} \frac{\beta_{t k}^{2}}{2 \Lambda_{t k}^{2}}\right) \\
\pi\left(\xi \mid \tau^{2}\right) \sim I G\left(1,1+\frac{1}{\tau^{2}}\right)
\end{array}
$$

The conditional density of the hyper-parameters in case of DL prior is

$$
\begin{array}{r}
\pi\left(\psi \mid \phi, \tau, \beta_{k}\right) \sim I G\left(\frac{\phi_{t k} \tau}{\left|\beta_{t k}\right|}, 1\right) \\
\pi\left(\tau \mid \phi, \beta_{k}\right) \sim G I G\left(p a-p, 1,2 \sum_{j=1}^{p} \frac{\left|\beta_{t k}\right|}{\phi_{t k}}\right) .
\end{array}
$$

To sample $\pi\left(\phi \mid \beta_{k}\right)$ sample $T_{t k} \sim G I G\left(a-1,1,2\left|\beta_{t k}\right|\right)$, set $\phi_{t k}=\frac{T_{t k}}{T}, T=$ $\sum_{t=1}^{p} T_{t k}$. where $\operatorname{GIG}(a, b, c)$ is the Generalized Inverse Gaussian distribution with density $f(x ; a, b, c) \propto x^{(c-1)} e^{\frac{-1}{2}\left(a x+\frac{b}{x}\right)}$.

The conditional density of the hyper-parameters are as follows:

$$
\begin{array}{r}
\pi\left(\tau_{t k} \mid \beta_{k}, \Lambda, y\right) \sim \operatorname{GIG}\left(\frac{1}{2}, \Lambda_{t k}^{2}, \beta_{t k}^{2}\right) \\
\pi\left(\Lambda \mid \beta_{k}, y\right) \sim \operatorname{Gamma}\left(\zeta+1, \eta+\left|\beta_{t k}\right|\right) .
\end{array}
$$

Here, $\beta_{k}=\left(\beta_{1 k}, \beta_{2 k}, \ldots, \beta_{p k}\right), t=1,2, \ldots, p$.

\section{Simulation and Results}

Data is simulated from a logistic model under various settings of sample sizes $(n)$, dimensions $(p)$, and effect sizes $\beta=\left(\beta_{1}, \beta_{2}, \ldots, \beta_{p}\right)$. The following measures were used for assessing the quality of our prediction and VS. Accuracy (the proportion of correctly classified responses in the test dataset); Sensitivity ( the proportion of true positives ); Specificity ( the proportion of true negatives ); AUC (Area Under the Receiver Operating Characteristic (ROC) Curve ); Brier Score (BS) $B S=\frac{1}{N} \sum_{i=1}^{N}\left(P_{i}-Y_{i}\right)$; here, $P_{i}$ is the probability of prediction and $Y_{i}$ is the actual outcome at that instance. The best score achievable is 0 and the worst is 1 ; $\mathrm{L} 1$ error $=\frac{1}{p} \sum_{i=1}^{p}\left|\beta_{i}-\hat{\beta}_{i}\right| ; \mathrm{L} 2$ error $=\sqrt{\frac{1}{p} \sum_{i=1}^{p}\left(\beta_{i}-\hat{\beta}_{i}\right)^{2}} ;$ Mean Squared Error (MSE) can be obtained by (L2error $)^{2}$. It is expected to have higher values for Accuracy, Sensitivity, Specificity, AUC, and lower values for BS, L1 error, and L2 error. The description of the evaluation metrics is obtained from a confusion matrix. A total of 100 data sets were simulated for each of the conditions. All the Gibbs sampling steps are written in $\mathrm{R}$ [37]. The length of the Markov Chain Monte Carlo (MCMC) simulation is 10000, and the burn-in size is 6000 iterations, which 
are discarded. Seven simulation scenarios are considered. There is an $80-20$ partition in the training and test data sets. Cross-validation for data partition did not provide substantial performance enhancement. Table 1 summarizes the simulation results and simulation parameters for prediction and VS. It has five and six panels containing the values of the evaluation metrics defined above. Each panel has three rows corresponding to the three prior settings. Figure 1 gives the VS and prediction performance concerning accuracy, L1 error, and BS across the seven simulation scenarios.

BS1 and BS2 have similar simulation settings except for a change of sample size, and it shows that the prediction measures decrease and BS increases from BS1 to BS2. BS1 is an ideal simulated data situation that achieves $100 \%$ sensitivity, can determine all the non-zero coefficients. BS3, BS4, and BS5 have a different set of coefficients. In BS3 and BS4, the decrease in the $N / P$ ratio and $\%$ of non-zero $\beta$ lowers the respective prediction measures, despite a low correlation. For VS, there is a clear demarcation from BS3 to BS4, and the effect sizes of BS4 can be identified easily that BS3. From BS3 and BS5, the $N / P$ ratio decrease, and a high correlation at such a small sample size decreases the prediction \& VS measures. For BS6, the $N / P$ ratio and $\%$ of non-zero beta are meager, and also, there is a high correlation which does not affect prediction; however, the VS suffers in identifying the non-zero coefficients though it can identify all the zero coefficients. There is an anomaly in the specificity in some scenarios since the control type-I and type-II error of a hypothesis cannot be carried out simultaneously. All three priors perform uniformly, so the method is robust to prior choices. The data is generated from the MLR model under varied sample size $N$, variable size $P$, and coefficients beta. The covariates are obtained with mean $m$ and $s d=1$. In all the simulation scenarios, the demarcation between training and test sets is in the ration of 80:20, and the Jth category is the reference class. The measures for prediction performance are Accuracy and AUC. The simulation parameters, prediction, and variable selection measures are in Table 2 and can be well distinguished from figure 3. Comparing MS1 and MS2, the sample size decrease does not affect prediction, though all variable selection measures suffer from it. An increase in the number of variables has a significant impact when MS3 is compared to MS1 in prediction and variable importance. MS4 has $N / P=33.33$ with $100 \%$ identification of non-zero coefficients, maximum accuracy, and AUC in prediction, but MSE, L1 error, and L2 error, especially for Horseshoe, DP are high concerning other simulation scenarios. Though the coefficients and $N / P$ ratio changes from MS4 to MS5, the algorithm can obtain optimum prediction measures and performs well in coefficient identification. The increases in no. of classes have an impact in reducing the accuracy in MS6. Scenarios like MS7 need further exploration; it depends on the $N / P$ ratio and the coefficients and performs reasonably here. In all the scenarios, the standard deviation among data sets is highest for DL prior, especially for VS. There is not a single prior; different scenarios have different sets of prior performing better than the others.

\section{Data Application}

The method is validated by applying it to standard data examples. For all the case studies, MCMC burn-in size is 6000, and no. of MCMC iterations is 10000. The training and test set size is of ratio 80/20. All the data description is in Table 3 . 


\section{Pima Indians Diabetes}

The method is validated by applying it to standard data examples. For all the case studies, MCMC burn-in size is 6000, and no. of MCMC iterations is 10000 . The training and test set size is of ratio 80/20. All the data description is in Table 3 . The data has been analyzed in the literature related to shrinkage priors, including $[38,39,40]$. The three priors can detect four variables by $95 \%$ credible intervals. The credible intervals of the coefficients with variable names "pregnant," "glucose," "mass (BMI)," and "pedigree (family history)" do not include zero; hence they are significant. These results are at par with [38]; however, the "pressure" variable is determined as significant only by DP prior. These three priors are also compared with Bayesian-Lasso(BLasso) [38], Bayesian-Elastic-Net(BElastic) [41] and frequentist methods like Lasso [6],

Elastic-Net [7] and Ridge [9]. Here, the three priors' accuracy is similar, but BS is the least among all the methods. Even though EN and Ridge have high accuracy, the frequentist methods have substantial-high BS and low specificity values.

\section{Colon}

This data first published in [42] comprises gene expression levels for 40 tumors and 22 normal colon tissues are measured for 6500 human genes using the Affymetrix oligonucleotide arrays. Samples are obtained from tumor tissue and adjacent, unaffected parts of the colon of the same patients [43]. Out of these, 2000 genes with the highest minimal intensity across the tissues are selected for classification purposes [42]. In the public domain, there is no demographic information available for the two groups like race, age, sex distribution; else, a study could have been conducted about the benefits of inclusion of one or all these covariates in prediction. Lasso selects 14 genes. The priors are applied to the data with the lasso estimates as the initial values. Here, we adopt a similar approach of k-means from [18] with a similar traditional prostate cancer dataset. We cluster the $\left|\beta_{i}\right|$ by K-means algorithm at each MCMC iteration with 2 clusters. For each iteration, the number of non-zero $\beta$ 's is then estimated by the smaller cluster size out of the two clusters. A final estimate $(\mathrm{F})$ of the number of non-zero signals is obtained by taking the highest frequency over all the MCMC iterations. We then trace back and find the F-number of gene IDs for each iteration and take the first $\mathrm{F}$ genes with the highest frequency. In the case of Horseshoe prior, it selects 36 genes with the highest frequencies, among which one gene (Gene ID: 1423) is included among the top 20 genes selected by the t-test and fold change [44]. Also, the Gene ID: 1325 selected by Lasso [45] can be seen in the set of 36 genes selected by Horseshoe. With a similar algorithm, Dirichlet Laplace selects 164 genes, of which Gene ID: 138 is included in the top 2 genes. DP selects 67 genes, out of which eight genes are in the set of genes selected by Horseshoe.The MCMC Gibbs sampling mixing and convergence were determined by Trace Plots and Auto-Correlation plots with an average adequate sample size of 23095.21 for Horseshoe, 39755.4 for DL, and 40129.35 for DP. About $95 \%$ of the genes conform to the Geweke Diagnostic criteria for all three priors. A thinning step at every 15 steps of iteration did not significantly improve the results, so we did a posterior prediction analysis on the 40,000 iterations after burn-in. The MSE for HS, DL, and DP are 0.001,0.003,0.003, implying that MCMC has converged well. Here, on the basis, BS Horseshoe prior performs better than the other priors. 
ADNI

Alzheimer's disease (AD) is a critical public health concern throughout the world and one of the most widespread neurodegenerative disorder [46]. AD is an irreparable brain disease, which impairs thinking and memory. Several machine learning methods have helped predict AD from Mild Cognitive Impairment (MCI); here, the shrinkage priors are used to predict $\mathrm{AD}$ from MCI, and they achieve high prediction measures. The data used here is from the R package ADNIMERGE [47] is a subset of the "adnimerge" data that contains only baseline variable measurements (i.e., the first visit for each participant) that has a diagnosis. The "adnimerge" dataset merges several key variables from various case report forms and biomarker lab from all ADNI protocols (ADNI1, ADNIGO, and ADNI2). The integrated data consists of 113 variables and 14712 observations, which include multiple observations per participant, representing multiple visits to the study's site for participant evaluation The participants is divided into five different classes namely Cognitively Normal $(\mathrm{CN}=4428)$, Early Mild Cognitive Impairment $($ EMCI $=2687)$, Late Mild Cognitive Impairment (LMCI = 4993), Subjective Memory Complaint (SMC $=938)$, and Alzheimer's Disease $(\mathrm{AD}=1654)$. After pre-processing, there were 22 variables, including the outcome. The demographic, cognitive assessment and clinical assessment attributes are in [48]. The missing values are discarded, and the data is normalized. The three priors, along with Bayesian lasso and Bayesian EN, are used to predict AD from MCI. The three priors achieve $85 \%$ accuracy and 93.5\% specificity for the prediction of $\mathrm{AD}$ from the MCI stage and have comparable results with other shrinkage priors. The results also indicate that the most distinguishing attribute for the prediction of AD includes the CDRSB.bl, ADAS13.bl, RAVLT.perc.forgetting.bl cognitive test among the cognitive assessment attributes, the volume of the whole brain among the patient's clinical assessment attributes and age among the demographic attributes.

\section{OASIS}

This data set consists of a longitudinal collection of Non-demented and Demented Older Adults, 150 subjects aged 60 to 96. The three priors' most frequently included variables are Age, SES, MMSE, gender, ASF, and nWBV. The three priors' prediction efficiency shows that they outperform other methods concerning BS and specificity. Table 4 , and the circular bar chart figure 2 gives a detailed view of the prediction performance in LR model. Figure 2 is efficient in describing the comparison of all the four data sets in a single platform. For each data set, eight bars correspond to the eight shrinkage methods, and each stacked bar consists of the five measures of prediction. For ADNI and PIMA all the methods perform similarly, though the priors do not prove to be efficient in the high-dimensional Colon data. For the MLR model, in ADNI data, the three classes are considered as EMCI, LMCI, and AD, and collected data for these classes had a dimension of $1438 \times 113$. A 10- fold cross-validation was used for prediction analysis. The variables selected by the priors are "CDRSB.bl," "MMSE.bl," "WholeBrain.bl", "Fusiform.bl", and "ICV," which are real-life markers for AD prediction and being validated by previous literature. The five-category model did not have a high performance in terms of accuracy. DL and DP provide better metrics than Horseshoe here. For OASIS 
data, the categorical response variable is classified into three classes. The moderate (1) and mild CDR (2) ratings are combined since there were only four observations for the moderate class. The variables selected are "Age","Education","SES", "MMSE", and "nWBV". Though Horseshoe and DP have high accuracy, DL may be a better choice for this data due to high cross-entropy and values DL. Accuracy and AUC are presented in parenthesis for the three priors in Table 4. Figure 4 is prepared by the R packages HUM [49], and plotly [50] that provides detail about the behavior of the shrinkage priors and proves that selected variables and volume under the surface are the same. The ROC surface for Horseshoe prior is presented here; all the other priors follow similarly.

\section{Discussion}

Bayesian shrinkage models can be utilized as a practical and useful alternative classification approach and a plausible way to select genetic markers and risk factors. This is a detailed study with a wide variety of settings comparing the three most well-known shrinkage priors. All of the three priors were able to recognize patterns differentiating the binary classes to a highly accurate level. This was shown by the excellent predictive performance of the binary logistic regression model on the simulated data sets and fairly high predictive accuracies on a wide array of public health data sets. Some of these datasets were super high dimensional which exhibits the model's power to scale up to these challenging scenarios. The algorithm is efficient, and the time taken to execute the simulations is relatively low such as algorithm with $n=500 ; p=50$ takes about 673 seconds. This combination of computational power and predictive performance makes it a very reasonable method of use for practitioners who require quick high dimensional analysis retaining the advantages of Bayesian analysis. In future we would formally explore the comparison with priors like Spike and Slab prior and schemes like the MH algorithm. We posit that features like selections of indicators and acceptance-rejection step would not favorably compare with the proposed algorithm We also extended the model to a multinomial logistic model that handles multiple categories. An R package "ShrinkageBayesGlm" is developed to be publicly available soon. We expect that coming years will witness its wider dissemination among public health research, and will be useful for predicting occurrences of common disorders like dementia, colon cancer, diabetes etc. Computational advances, especially in high-dimensional case [51] will continue expanding the scope of exact methods.

Acknowledgements

Authors would like to acknowledge Dr. Craig McClain and Dr. S. Srivastava for the funding to conduct the research.

ADNI data

Data collection and sharing for the ADNI data application in this article was funded by the Alzheimer's Disease Neuroimaging Initiative (ADNI) (National Institutes of Health Grant U01 AG024904) and DOD ADNI (Department of Defense award number W81XWH-12-2-0012). ADNI is funded by the National Institute on Aging, the National Institute of Biomedical Imaging and Bioengineering, and through generous contributions from the following: AbbVie, Alzheimer's Association; Alzheimer's Drug Discovery Foundation; Araclon Biotech; BioClinica, Inc.; Biogen; Bristol-Myers Squibb Company; CereSpir, Inc.; Cogstate; Eisai Inc.; Elan Pharmaceuticals, Inc.; Eli Lilly and Company; Eurolmmun; F. Hoffmann-La Roche Ltd and its affiliated company Genentech, Inc.; Fujirebio; GE Healthcare; IXICO Ltd.; Janssen Alzheimer Immunotherapy Research \& Development, LLC.; Johnson \& Johnson Pharmaceutical Research \& Development LLC.; Lumosity; Lundbeck; Merck \& Co., Inc.; Meso Scale Diagnostics, LLC.; NeuroRx Research; Neurotrack Technologies; Novartis Pharmaceuticals Corporation; Pfizer Inc.; Piramal Imaging; Servier; Takeda Pharmaceutical Company; and Transition Therapeutics. The Canadian Institutes of Health 
Research is providing funds to support ADNI clinical sites in Canada. Private sector contributions are facilitated by the Foundation for the National Institutes of Health (www.fnih.org). The grantee organization is the Northern California Institute for Research and Education, and the study is coordinated by the Alzheimer's Therapeutic Research Institute at the University of Southern California. ADNI data are disseminated by the Laboratory for Neuro Imaging at the University of Southern California.

\section{OASIS data}

Authors would like to acknowledge for the following for the OASIS data

- OASIS: Cross-Sectional: Principal Investigators: D. Marcus, R, Buckner, J, Csernansky J. Morris; P50 AG05681, P01 AG03991, P01 AG026276, R01 AG021910, P20 MH071616, U24 RR021382

- OASIS: Longitudinal: Principal Investigators: D. Marcus, R, Buckner, J. Csernansky, J. Morris; P50 AG05681, P01 AG03991, P01 AG026276, R01 AG021910, P20 MH071616, U24 RR021382

- OASIS-3: Principal Investigators: T. Benzinger, D. Marcus, J. Morris; NIH P50AG00561, P30NS09857781, P01AG026276, P01AG003991, R01AG043434, UL1TR000448, R01EB009352. AV-45 doses were provided by Avid Radiopharmaceuticals, a wholly owned subsidiary of Eli Lilly.

\section{Funding}

This work was supported by the National Institute of Health grant P42 ES023716 to principal investigator: Dr S Srivastava and the National Institute of Health grant 1P20 GM113226 to principal investigator: Dr C McClain. Dr. Shesh N. Rai was also partially supported by Wendell Cherry Chair in Clinical Trial Research.

\section{Abbreviations}

SSVS - Stochastic Search Variable Selection; GL- global-local; HS- Horseshoe; DL- Dirichlet Laplace; DP - Double Pareto; PG - Polya-Gamma; EN - Elastic Net; BE - Bayesian Elastic Net; BL - Bayesian Lasso; LR - Logistic Regression; MLR - Multinomial Logistic Regression; DA - Data-Augmentation; MCMC- Markov Chain Monte Carlo; AUC- Area Under Curve; ROC- Receiver Operating Characteristic Curve; MSE - Mean Squared Error; BS - Brier Score; VS - variable selection;

\section{Availability of data and materials}

The datasets used and/or analysed during the current study are available and information about it is included in this article in Table 3.

Ethics approval and consent to participate

Not applicable

Competing interests

The authors declare that they have no competing interests.

\section{Consent for publication}

Not applicable

Authors' contributions

A.B. has contributed to the methodology, data collection, analysis, and writing of the manuscript. S.P. and R.M have contributed equally to methodology and analysis. S.N.R. has contributed to developing ideas and valuable comments. All authors have contributed to the final preparation of the manuscript.

\section{Author details}

${ }^{1}$ Department of Bioinformatics \& Biostatistics, University of Louisville, KY, USA. ${ }^{2}$ Biostatistics \& Bioinformatics Facility, JG Brown Cancer Center, University of Louisville, KY, USA. ${ }^{3}$ The Christina Lee Brown Envirome Institute, University of Louisville, KY, USA. ${ }^{4}$ University of Louisville Alcohol Research Center, University of Louisville, KY, USA. ${ }^{5}$ University of Louisville Hepatobiology \& Toxicology Center, University of Louisville, KY, USA.

\section{References}

1. Reuter, J.A., Spacek, D.V., Snyder, M.P.: High-throughput sequencing technologies. Molecular cell 58(4), 586-597 (2015)

2. Newton, K., Newman, W., Hill, J.: Review of biomarkers in colorectal cancer. Colorectal disease 14(1), 3-17 (2012)

3. Krishnamurti, U., Silverman, J.F.: Her2 in breast cancer: a review and update. Advances in anatomic pathology 21(2), 100-107 (2014)

4. Cappuzzo, F., Gregorc, V., Rossi, E., Cancellieri, A., Magrini, E., Paties, C.T., Ceresoli, G., Lombardo, L., Bartolini, S., Calandri, C., et al.: Gefitinib in pretreated non-small-cell lung cancer (nsclc): Analysis of efficacy and correlation with her2 and epidermal growth factor receptor expression in locally advanced or metastatic nsclc. Journal of clinical oncology 21(14), 2658-2663 (2003)

5. Barnes, D.E., Lee, S.J.: Predicting alzheimer's risk: why and how? Alzheimer's research \& therapy 3(6), 33 (2011)

6. Tibshirani, R.: Regression shrinkage and selection via the lasso. Journal of the Royal Statistical Society: Series B (Methodological) 58(1), 267-288 (1996)

7. Zou, H., Hastie, T.: Regularization and variable selection via the elastic net. Journal of the royal statistical society: series B (statistical methodology) 67(2), 301-320 (2005)

8. Li, Y., Horowitz, M.A., Liu, J., Chew, A., Lan, H., Liu, Q., Sha, D., Yang, C.: Individual-level fatality prediction of covid-19 patients using ai methods. Frontiers in Public Health 8, 566 (2020)

9. Hoerl, A.E., Kennard, R.W.: Ridge regression: Biased estimation for nonorthogonal problems. Technometrics 12(1), 55-67 (1970) 
10. Bae, K., Mallick, B.K.: Gene selection using a two-level hierarchical bayesian model. Bioinformatics 20(18), 3423-3430 (2004)

11. Bhadra, A., Datta, J., Polson, N.G., Willard, B., et al.: The horseshoe+ estimator of ultra-sparse signals. Bayesian Analysis 12(4), 1105-1131 (2017)

12. Griffin, J., Brown, P., et al.: Hierarchical shrinkage priors for regression models. Bayesian Analysis 12(1), 135-159 (2017)

13. Ishwaran, H., Rao, J.S., et al.: Spike and slab variable selection: frequentist and bayesian strategies. The Annals of Statistics 33(2), 730-773 (2005)

14. Makalic, E., Schmidt, D.F.: A simple sampler for the horseshoe estimator. IEEE Signal Processing Letters 23(1), 179-182 (2015)

15. Park, T., Casella, G.: The bayesian lasso. Journal of the American Statistical Association 103(482), 681-686 (2008)

16. Piironen, J., Vehtari, A.: On the hyperprior choice for the global shrinkage parameter in the horseshoe prior. arXiv preprint arXiv:1610.05559 (2016)

17. Armagan, A., Dunson, D.B., Lee, J.: Generalized double pareto shrinkage. Statistica Sinica 23(1), 119 (2013)

18. Bhattacharya, A., Pati, D., Pillai, N.S., Dunson, D.B.: Dirichlet-laplace priors for optimal shrinkage. Journal of the American Statistical Association 110(512), 1479-1490 (2015)

19. Van Erp, S., Oberski, D.L., Mulder, J.: Shrinkage priors for bayesian penalized regression. Journal of Mathematical Psychology 89, 31-50 (2019)

20. Mitchell, T.J., Beauchamp, J.J.: Bayesian variable selection in linear regression. Journal of the American Statistical Association 83(404), 1023-1032 (1988)

21. George, E.I., McCulloch, R.E.: Variable selection via gibbs sampling. Journal of the American Statistical Association 88(423), 881-889 (1993)

22. George, E.I., McCulloch, R.E.: Approaches for bayesian variable selection. Statistica sinica, 339-373 (1997)

23. Green, P.J., Hastie, D.I.: Reversible jump mcmc. Genetics 155(3), 1391-1403 (2009)

24. Polson, N.G., Scott, J.G.: Shrink globally, act locally: Sparse bayesian regularization and prediction. Bayesian statistics 9, 501-538 (2010)

25. Griffin, J.E., Brown, P.J., et al.: Inference with normal-gamma prior distributions in regression problems. Bayesian Analysis 5(1), 171-188 (2010)

26. Carvalho, C.M., Polson, N.G., Scott, J.G.: The horseshoe estimator for sparse signals. Biometrika 97(2), 465-480 (2010)

27. O'Hara, R.B., Sillanpää, M.J., et al.: A review of bayesian variable selection methods: what, how and which Bayesian analysis 4(1), 85-117 (2009)

28. Albert, J.H., Chib, S.: Bayesian analysis of binary and polychotomous response data. Journal of the American statistical Association 88(422), 669-679 (1993)

29. Polson, N.G., Scott, J.G., Windle, J.: Bayesian inference for logistic models using pólya-gamma latent variables. Journal of the American statistical Association 108(504), 1339-1349 (2013)

30. Choi, H.M., Hobert, J.P., et al.: The polya-gamma gibbs sampler for bayesian logistic regression is uniformly ergodic. Electronic Journal of Statistics 7, 2054-2064 (2013)

31. Van Dyk, D.A., Meng, X.-L.: The art of data augmentation. Journal of Computational and Graphical Statistics 10(1), 1-50 (2001)

32. Hobert, J.P., Marchev, D., et al.: A theoretical comparison of the data augmentation, marginal augmentation and px-da algorithms. The Annals of Statistics 36(2), 532-554 (2008)

33. Holmes, C.C., Held, L., et al.: Bayesian auxiliary variable models for binary and multinomial regression. Bayesian analysis 1(1), 145-168 (2006)

34. Frühwirth-Schnatter, S., Frühwirth, R.: Data augmentation and mcmc for binary and multinomial logit models. In: Statistical Modelling and Regression Structures, pp. 111-132. Springer, ??? (2010)

35. Carvalho, C.M., Polson, N.G., Scott, J.G.: Handling sparsity via the horseshoe. Proceedings of the Twelth International Conference on Artificial Intelligence and Statistics 5 5, 73-80 (2009)

36. Bhattacharya, A., Chakraborty, A., Mallick, B.K.: Fast sampling with gaussian scale mixture priors in high-dimensional regression. Biometrika, 042 (2016)

37. R Core Team: R: A Language and Environment for Statistical Computing. R Foundation for Statistical Computing, Vienna, Austria (2020). R Foundation for Statistical Computing. https://www.R-project.org/

38. Makalic, E., Schmidt, D.F.: High-dimensional bayesian regularised regression with the bayesreg package. arXiv preprint arXiv:1611.06649 (2016)

39. Bové, D.S., Held, L., et al.: Hyper-g priors for generalized linear models. Bayesian Analysis 6(3), 387-410 (2011)

40. Ghosh, J., Li, Y., Mitra, R., et al.: On the use of cauchy prior distributions for bayesian logistic regression. Bayesian Analysis 13(2), 359-383 (2018)

41. Huang, A., Liu, D.: Ebglmnet: a comprehensive $r$ package for sparse generalized linear regression models. Bioinformatics (2016)

42. Alon, U., Barkai, N., Notterman, D.A., Gish, K., Ybarra, S., Mack, D., Levine, A.J.: Broad patterns of gene expression revealed by clustering analysis of tumor and normal colon tissues probed by oligonucleotide arrays. Proceedings of the National Academy of Sciences 96(12), 6745-6750 (1999)

43. Sakellariou, A., Sanoudou, D., Spyrou, G.: Combining multiple hypothesis testing and affinity propagation clustering leads to accurate, robust and sample size independent classification on gene expression data. BMC bioinformatics 13(1), 270 (2012)

44. Pepe, M.S., Longton, G., Anderson, G.L., Schummer, M.: Selecting differentially expressed genes from microarray experiments. Biometrics 59(1), 133-142 (2003)

45. Algamal, Z.: An efficient gene selection method for high-dimensional microarray data based on sparse logistic regression. Electronic Journal of Applied Statistical Analysis 10(1), 242-256 (2017)

46. Bäckman, L., Jones, S., Berger, A.-K., Laukka, E.J., Small, B.J.: Cognitive impairment in preclinical 
alzheimer's disease: a meta-analysis. Neuropsychology 19(4), 520 (2005)

47. the ADNI team: ADNIMERGE: Alzheimer's Disease Neuroimaging Initiative. (2020). R package version 0.0.1

48. Shahbaz, M., Ali, S., Guergachi, A., Niazi, A., Umer, A.: Classification of alzheimer's disease using machine learning techniques (2019)

49. Novoselova, N., Della Beffa, C., Wang, J., Li, J., Pessler, F., Klawonn, F.: Hum calculator and hum package for r: easy-to-use software tools for multicategory receiver operating characteristic analysis. Bioinformatics 30(11), 1635-1636 (2014)

50. Sievert, C.: Interactive Web-Based Data Visualization with R, Plotly, and Shiny. Chapman and Hall/CRC, ??? (2020). https://plotly-r.com

51. Lee, S.Y., Pati, D., Mallick, B.K.: Continuous shrinkage prior revisited: a collapsing behavior and remedy. arXiv preprint arXiv:2007.02192 (2020)

52. Leisch, F., Dimitriadou, E., Leisch, M.F., No, Z.: Package 'mlbench' (2009)

53. Silva, P.D.: Hidimda: An $r$ package for supervised classification of high-dimensional data. (2012)

54. Marcus, D.S., Fotenos, A.F., Csernansky, J.G., Morris, J.C., Buckner, R.L.: Open access series of imaging studies: longitudinal mri data in nondemented and demented older adults. Journal of cognitive neuroscience 22(12), 2677-2684 (2010)

\section{Figures}
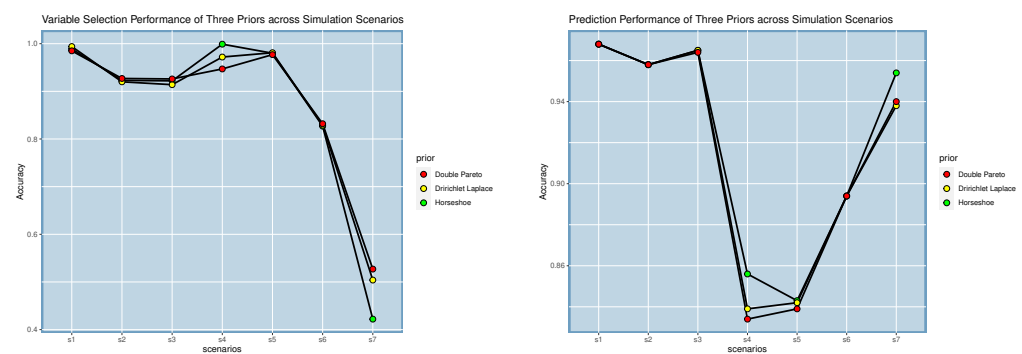

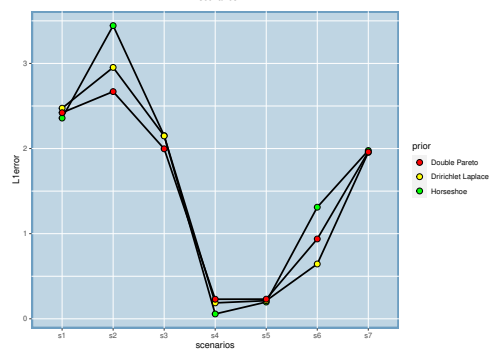

(a) Variable Selection Performance

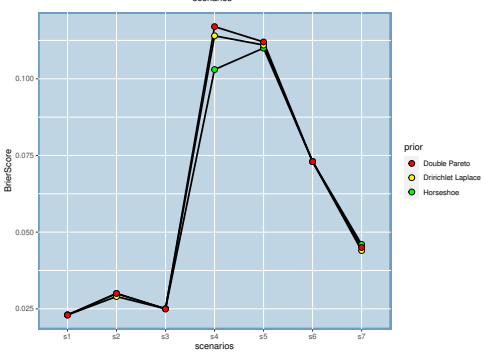

(b) Prediction Performance

Figure 1: Prediction \& Variable Selection Performance in LR with Shrinkage Priors across Simulation Scenarios 


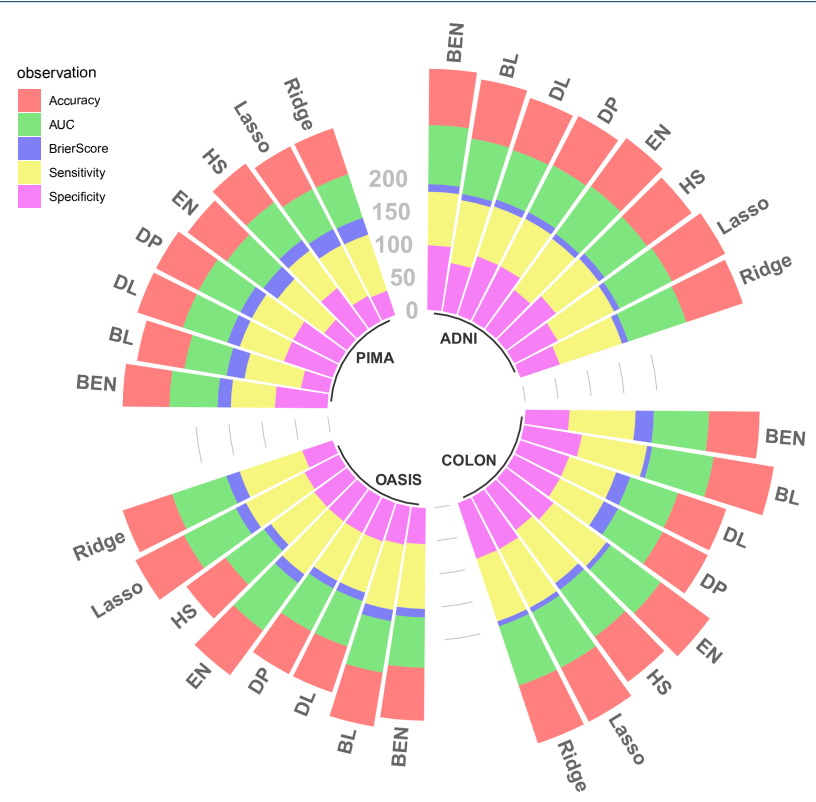

Figure 2: Circular Bar Chart comparing Prediction Metrics among datasets

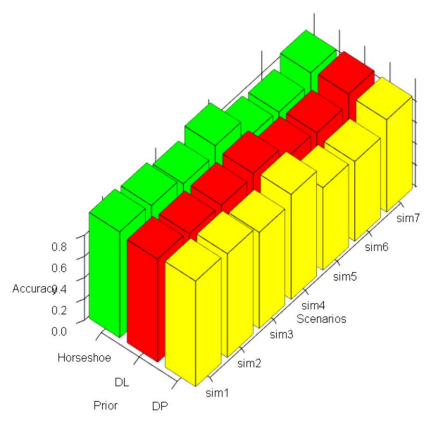

(a) Variable Selection Performance

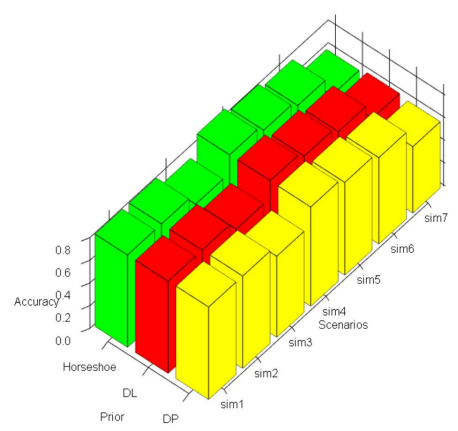

(b) Prediction Performance

Figure 3: Prediction \& Variable Selection Performance in MLR with Shrinkage Priors across Simulation Scenarios 


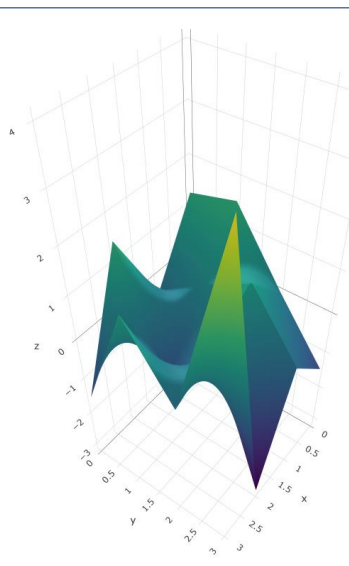

Figure 4: ROC Surface Plot for Horseshoe prior in data sets

Table 1: Prediction \& Variable Selection Performance for LR with Shrinkage Priors.

\begin{tabular}{|c|c|c|c|c|c|c|c|}
\hline & Predictior & & & & & & \\
\hline 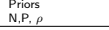 & $\begin{array}{l}\text { BSI } \\
1000,10,0.5 \\
\end{array}$ & $\begin{array}{l}\text { BS2 } \\
200,10,0.5 \\
\end{array}$ & $\begin{array}{l}\text { BS33. } \\
400,20,0.5 \\
\end{array}$ & $\begin{array}{l}\text { BSS4 } \\
500,50,0.3 \\
\end{array}$ & $\begin{array}{l}\text { BSS } \\
300,10,0.5 \\
\end{array}$ & $\begin{array}{c}\text { BSB6 } \\
100,10,0.5 \\
\end{array}$ & $\begin{array}{l}1557,0.5 \\
100,130,0.5 \\
\end{array}$ \\
\hline$\beta$ & $(10,10,10,10,5,5,0.1,0.1,0.1,0.1)^{\prime}$ & $(10,10,10,10,5,5,0.1,0.1,0.1,0.1)^{\prime}$ & $(\underbrace{10, \ldots, 10,}_{5} \underbrace{5, \ldots, 5,5}_{5} \underbrace{0.1, \ldots, 0.1}_{10})$ & $40 \%$ non-zero $\beta$ & $(1,1,1.5,-2,2,5,0,0,0,0,0,0,0,0)^{\prime}$ & $\begin{array}{c}(5,5,3,0,0.74,-0.9,0,0,0,0,0,0)^{\prime} \\
\end{array}$ & $(\underbrace{5, \ldots, 5, \underbrace{5}, \underbrace{0, \ldots, 5}_{100}}_{30})$ \\
\hline $\begin{array}{l}\text { Accuracy } \\
\text { Horseshoe } \\
\text { Dirichlet Laplace } \\
\text { Double Pareto }\end{array}$ & $\begin{array}{l}0.968(0.012) \\
0.968(0.012) \\
0.968(0.012)\end{array}$ & $\begin{array}{l}0.958(0.031) \\
0.958(0.030) \\
0.958(0.031)\end{array}$ & $\begin{array}{l}0.965(0.021) \\
0.965(0.020) \\
0.964(0.021)\end{array}$ & $\begin{array}{l}0.856(0.032) \\
0.839(0.039) \\
0.834(0.040)\end{array}$ & $\begin{array}{l}0.843(0.048) \\
0.84(2.050) \\
0.839(0.050)\end{array}$ & $\begin{array}{l}0.894(0.074) \\
0.894(0.075) \\
0.894(0.073)\end{array}$ & $\begin{array}{l}0.954(0.053) \\
0.938(0.062) \\
0.940(0.050)\end{array}$ \\
\hline $\begin{array}{l}\text { Horseshoe } \\
\text { Dirichlet Laplace } \\
\text { Double Pareto } \\
\end{array}$ & $\begin{aligned} \text { Sensitivity } \\
0.967(0.016) \\
0.967(0.016) \\
0.967(0.016) \\
\end{aligned}$ & $\begin{array}{l}0.965(0.043) \\
0.964(0.043) \\
0.964(0.044)\end{array}$ & $\begin{array}{l}0.964(0.031) \\
0.964(0.031) \\
0.964(0.030)\end{array}$ & $\begin{array}{l}0.853(0.047) \\
0.840(0.053) \\
0.835(0.055)\end{array}$ & $\begin{array}{l}0.059(0.030) \\
0.840(0.067) \\
0.837(0.068) \\
0.835(0.067)\end{array}$ & 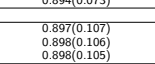 & $\begin{array}{l}0.940(0.005) \\
0.940(0.076) \\
0.944(0.073) \\
0.946(0.073)\end{array}$ \\
\hline $\begin{array}{l}\text { Horseshoe } \\
\text { Dirichlet Laplace } \\
\text { Double Pareto } \\
\end{array}$ & $\begin{array}{r}\text { Specificity } \\
0.9690 .017) \\
0.0690(0.017) \\
0.969(0.017) \\
\end{array}$ & $\begin{array}{l}0.953(0.046) \\
0.955(.046) \\
0.954(0.045)\end{array}$ & $\begin{array}{l}0.904(0.030) \\
0.966(0.031) \\
0.967(0.028) \\
0.964(0.030)\end{array}$ & $\begin{array}{l}0.055(0.055) \\
0.8590 .052) \\
0.838(0.058) \\
0.833(0.058)\end{array}$ & $\begin{array}{l}0.053(0.007) \\
0.847(0.070) \\
0.847(0.070) \\
0.847(0.070)\end{array}$ & $\begin{array}{l}0.090(0.105) \\
0.893(0.094) \\
0.8990(.097) \\
0.893(0.094)\end{array}$ & $\begin{array}{l}0.940(0.07) \\
0.943(0.084) \\
0.935(0.079) \\
0.934(0.079)\end{array}$ \\
\hline $\begin{array}{l}\text { Horseshoe } \\
\text { Dirichlet Laplace } \\
\text { Double Pareto } \\
\end{array}$ & $\begin{array}{r}\text { Area Under Curve } \\
0.968(0.012) \\
0.968(0.012) \\
0.968(0.012)\end{array}$ & $\begin{array}{l}0.958(0.031) \\
0.958(0.029) \\
0.958(0.030)\end{array}$ & $\begin{array}{l}0.965(0.021) \\
0.966(0.020) \\
0.964(0.021)\end{array}$ & $\begin{array}{l}0.856(0.032) \\
0.839(0.039) \\
0.834(0.040)\end{array}$ & $\begin{array}{l}0.844(0.049) \\
0.84(2.050) \\
0.849(0.050)\end{array}$ & $\begin{array}{l}0.898(0.074) \\
0.897(0.075) \\
0.897(0.073)\end{array}$ & $\begin{array}{l}0.944(0.052) \\
0.939(0.063) \\
0.941(0.051)\end{array}$ \\
\hline $\begin{array}{l}\begin{array}{l}\text { Horseshoe } \\
\text { Dirichlet Laplace } \\
\text { Double Pareto }\end{array} \\
\end{array}$ & 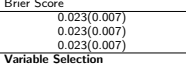 & $\begin{array}{l}0.030 .0 .018) \\
0.029(0.016) \\
0.030(0.017)\end{array}$ & $\begin{array}{l}0.025(0.013) \\
0.02(50.011) \\
0.025(0.012)\end{array}$ & $\begin{array}{l}0.103(0.019) \\
0.114(4.022) \\
0.117(0.023)\end{array}$ & $\begin{array}{l}0.110(0.026) \\
0.111(0.026) \\
0.112(0.026)\end{array}$ & $\begin{array}{l}0.073(0.042) \\
0.07(30.041) \\
0.073(0.043)\end{array}$ & $\begin{array}{l}0.046(0.026) \\
0.044(0.026) \\
0.045(0.024)\end{array}$ \\
\hline $\begin{array}{l}\text { Horseshoe } \\
\text { Dirichlet Laplace } \\
\text { Double Pareto } \\
\end{array}$ & $\begin{array}{l}0.989(0.035) \\
0.994(0.024) \\
0.985(0.039)\end{array}$ & $\begin{array}{l}0.923(0.072) \\
0.920(0.070) \\
0.927(0.071)\end{array}$ & $\begin{array}{l}0.922(0.053) \\
0.9414(0.049) \\
0.926(0.052)\end{array}$ & $\begin{array}{l}0.999(0.004) \\
0.992(0.024) \\
0.947(0.034)\end{array}$ & $\begin{array}{l}0.980 .043) \\
0.981(0.044) \\
0.977(0.047)\end{array}$ & $\begin{array}{l}0.827(0.066) \\
0.829(0.064) \\
0.832(.071)\end{array}$ & $\begin{array}{l}0.422(0.262) \\
0.504(27275) \\
0.527(0.274)\end{array}$ \\
\hline $\begin{array}{l}\text { Horseshoe } \\
\text { Dirichlet Laplace } \\
\text { Double Pareto } \\
\end{array}$ & $\begin{array}{l}\text { 1. } \\
1.0000(0.000) \\
1.000(0.000) \\
1.000(0.000)\end{array}$ & $\begin{array}{l}0.885(0.13) \\
0.86(113) \\
0.882(0.112) \\
0.82)\end{array}$ & $\begin{array}{l}0.860(0.096) \\
0.83(0.093) \\
0.864(0.096)\end{array}$ & $\begin{array}{l}0.998(0.025) \\
0.998(0.025) \\
1.000(0.000)\end{array}$ & $\begin{array}{l}0.962(0.090) \\
0.978(0.072) \\
0.978(0.072)\end{array}$ & $\begin{array}{l}0.6620 .123) \\
0.668(0.114) \\
0.682(0.121)\end{array}$ & $\begin{array}{l}0.163(0.094) \\
0.13(0.096) \\
0.129(0.096)\end{array}$ \\
\hline $\begin{array}{l}\text { Horseshoe } \\
\text { Dirichlet Laplace } \\
\text { Double Pareto }\end{array}$ & $\begin{array}{l}\text { ty } \\
0.99220 .0006) \\
0.985(0.060) \\
0.962(0.096)\end{array}$ & $\begin{array}{l}0.980(0.068) \\
0.998(0.025) \\
0.995(0.035)\end{array}$ & $\begin{array}{l}0.985(0.039) \\
0.989(0.031) \\
0.988(0.033)\end{array}$ & $\begin{array}{l}.999(0.004) \\
0.969(0.026) \\
0.942(0.037)\end{array}$ & $\begin{array}{l}0.992(0.037) \\
0.983(0.058) \\
0.977(0.050)\end{array}$ & $\begin{array}{l}0.992(0.039) \\
0.990(0.044) \\
0.982(0.058)\end{array}$ & $\begin{array}{l}\quad \begin{array}{l}0.999(0.004) \\
0.999(.003) \\
0.999(0.002)\end{array} \\
0\end{array}$ \\
\hline $\begin{array}{l}\text { Horseshoe } \\
\text { Dirichlet Laplace } \\
\text { Double Pareto }\end{array}$ & $\begin{array}{l}2.358(0.417) \\
2.474(0.326) \\
2.421(0.387)\end{array}$ & $\begin{array}{l}3.444(3.895) \\
2.953(3.388) \\
2.669(0.601)\end{array}$ & $\begin{array}{l}2.152(1.194) \\
2.1448 .0329) \\
1.997(0.438)\end{array}$ & $\begin{array}{l}0.057(0.017) \\
0.187(0.045) \\
0.230(0.053)\end{array}$ & $\begin{array}{l}0.197(0.064) \\
0.213(0.073) \\
0.231(0.076)\end{array}$ & $\begin{array}{l}1.311(1.776) \\
0.644(289) \\
0.938(0.720)\end{array}$ & $\begin{array}{l}1.977(0.229) \\
1.955(220) \\
1.960(0.237)\end{array}$ \\
\hline $\begin{array}{l}\text { Horseshoe } \\
\text { Dirichlet Laplace } \\
\text { Double Pareto }\end{array}$ & $\begin{array}{l}3.063(0.572) \\
3.231(1.0466) \\
3.146(0.529)\end{array}$ & $\begin{array}{l}4.435(4.931) \\
3.849(0.507) \\
3.469(0.772)\end{array}$ & $\begin{array}{l}2.971(1.722) \\
3.015(5.585) \\
2.781(0.459)\end{array}$ & $\begin{array}{l}0.102(0.036) \\
0.277(0.081) \\
0.330(0.092)\end{array}$ & $\begin{array}{l}0.259(0.091) \\
0.27(0.103) \\
0.293(0.106)\end{array}$ & $\begin{array}{l}1.942(2.797) \\
0.896(0.456) \\
1.359(1.176)\end{array}$ & $\begin{array}{l}2.562(0.299) \\
2.582(2.293) \\
2.594(0.333)\end{array}$ \\
\hline
\end{tabular}

Table 2: Prediction Performance \& Variable Selection for MLR with Shrinkage Priors.

\begin{tabular}{|c|c|c|c|c|c|c|c|}
\hline 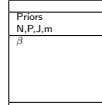 & 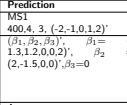 & 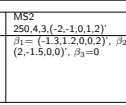 & 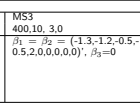 & 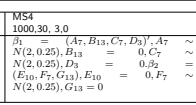 & 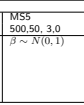 & 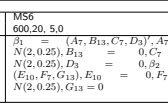 & 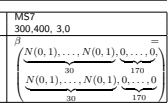 \\
\hline 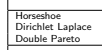 & 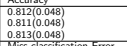 & 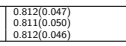 & 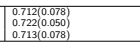 & 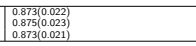 & 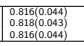 & 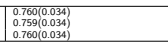 & 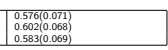 \\
\hline 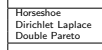 & 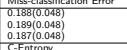 & 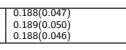 & 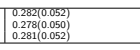 & 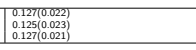 & 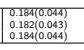 & 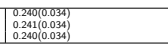 & 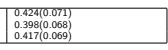 \\
\hline 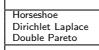 & 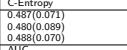 & 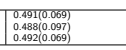 & 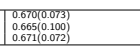 & 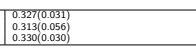 & 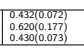 & 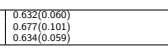 & 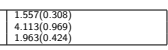 \\
\hline 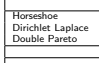 & 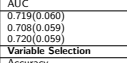 & 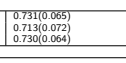 & 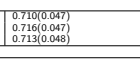 & 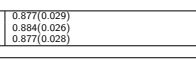 & 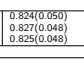 & 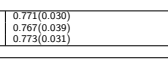 & 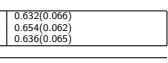 \\
\hline 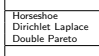 & 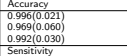 & 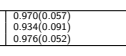 & 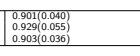 & 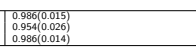 & 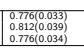 & 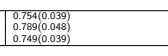 & 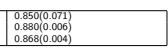 \\
\hline 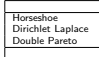 & 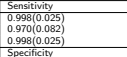 & 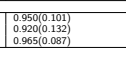 & 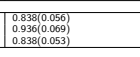 & 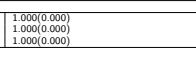 & 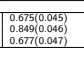 & 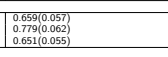 & 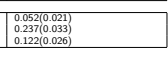 \\
\hline 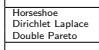 & 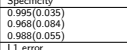 & 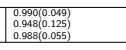 & 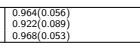 & 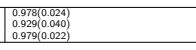 & 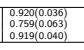 & 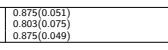 & 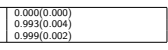 \\
\hline 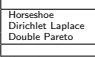 & 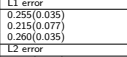 & 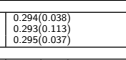 & 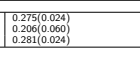 & 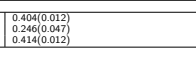 & 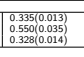 & 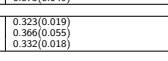 & 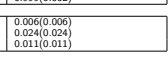 \\
\hline 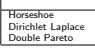 & 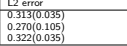 & 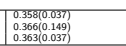 & 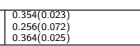 & 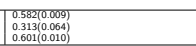 & 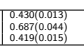 & 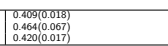 & 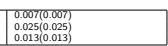 \\
\hline
\end{tabular}


Table 3: Data Description.

\begin{tabular}{|c|c|c|c|c|}
\hline Data & Availability & $\mathrm{N}, \mathrm{P}$ & \begin{tabular}{|l} 
Variables \\
\end{tabular} & Outcome \\
\hline Pima Indians Diabetes & R package "mlbench" [52] & 768,8 & $\begin{array}{l}\text { no. of times pregnant }\left(x_{1}\right), \\
\text { glucose }(x 2), \text { pressure }\left(x_{3}\right), \text { tri- } \\
\text { ceps: Triceps skin fold thickness } \\
(\mathrm{mm})\left(x_{4}\right), \text { insulin }\left(x_{5}\right), \quad \text { BMI } \\
\left(x_{6}\right) \text {, pedigree }\left(x_{7}\right), \operatorname{Age}\left(x_{8}\right)\end{array}$ & $\begin{array}{l}\text { tested positive for diabetes } \\
(y=1) \text {, negative }(y=0)\end{array}$ \\
\hline Colon & $\begin{array}{l}\text { R package like 'HiDimDA' } \\
\text { [53] }\end{array}$ & 62,2000 & human genes & $\begin{array}{l}40 \text { tumor }(y=1) \& 22 \text { nor- } \\
\text { mal }(y=0) \text { colon tissues }\end{array}$ \\
\hline ADNI & $\begin{array}{l}\text { Alzheimer's Disease } \mathrm{Neu}- \\
\text { roimaging Initiative (ADNI) } \\
\text { database (adni.loni.usc.edu) } \\
\mathrm{R} \text { package ADNIMERGE } \\
{[47]}\end{array}$ & $\begin{array}{l}\text { 14712, } 113, \text { after pre- } \\
\text { processing: } 911,22\end{array}$ & $\begin{array}{l}\text { Age, CDRSB_bl: Clinical De- } \\
\text { mentia Rating Sum of Boxes } \\
\text { (core), ADAS11_bl: 11 item- } \\
\text { AD Cognitive Scale (score), } \\
\text { ADAS13_bl: } 13 \text { item-AD Cog- } \\
\text { nitive Scale (score), MMSE_bl: } \\
\text { Mini-Mental State Examination } \\
\text { (score), RAVLT_immediate_bl, } \\
\text { RAVLT_learning_bl, } \\
\text { RAVLT_forgetting_bl, } \\
\text { RAVLT_perc_forgetting_bl: } \\
\text { Rey's Auditory Verbal Learning } \\
\text { Test (scores for immediate } \\
\text { response, learning, forgetting } \\
\text { and percentage forgetting), } \\
\text { FAQ_bl: Functional Activities } \\
\text { Questionnaire, APOE4: APOE4 } \\
\text { gene presence, Hippocampus_bl: } \\
\text { Volume of hippocampus, Ven- } \\
\text { tricles_bl: Volume of ventricles, } \\
\text { WholeBrain_bl: volume of Brain, } \\
\text { Fusiform_bl: The volume of the } \\
\text { fusiform gyrus, Entorhinal-bl: } \\
\text { The volume of the entorhinal } \\
\text { cortex, MidTemp_bl: The vol- } \\
\text { ume of the middle temporal } \\
\text { gyrus, ICV: Intra Cranial Vol- } \\
\text { ume, PTGENDER: Participant's } \\
\text { umender, PTETHCAT: Partici- } \\
\text { pant's ethicity, PTRACCAT: } \\
\text { Participant's race, PTMARRY: } \\
\text { Participant's marital status }\end{array}$ & $\begin{array}{l}\text { LR model: } \mathrm{AD}(y=1), \\
\mathrm{EMCl} \text { or } \mathrm{LMCl}(y=0) ; \mathrm{MLR} \\
\text { model: } \mathrm{AD}(y=2), \mathrm{EMCl} \\
(y=1), \operatorname{LMCl}(y=0)\end{array}$ \\
\hline OASIS & $\begin{array}{l}\text { Open Access Series of Imag- } \\
\text { ing Studies (OASIS) [54] }\end{array}$ & $\begin{array}{l}373, \quad 15 ; \quad \text { after } \\
\text { processing } 373,8\end{array}$ & $\begin{array}{l}\text { Visit: number of visits, gender, } \\
\text { age, EDUC: Education, SES: So- } \\
\text { cioeconomic status as assessed } \\
\text { by the Hollings head Index of } \\
\text { Social Position, MMSE: Mini- } \\
\text { Mental State Examination score, } \\
\text { nWBV: Normalized whole brain } \\
\text { volume, ASF: Atlas Scaling Fac- } \\
\text { tor }\end{array}$ & $\begin{array}{l}\text { CDR: Clinical Dementia Rat- } \\
\text { ing }(0=\text { no dementia, } 0.5= \\
\text { very mild } \mathrm{AD}, 1=\text { mild } \mathrm{AD}, \\
2=\text { moderate } \mathrm{AD}) ; \text { For } \mathrm{LR} \\
\text { model: mild } \mathrm{AD} \text { or } \mathrm{AD}(y= \\
1), \text { no dementia or very mild } \\
\mathrm{AD}(y=0) ; \text { For MLR model: } \\
\text { mild } \mathrm{AD} \text { or } \mathrm{AD}(y=2), \text { no } \\
\text { dementia }(y=0) \text {, very mild } \\
\mathrm{AD}(y=1)\end{array}$ \\
\hline
\end{tabular}

Table 4: Prediction Performance in Real Life Data for LR.

\begin{tabular}{|c|c|c|c|c|c|c|c|c|}
\hline \multicolumn{9}{|c|}{ Priors } \\
\hline Measures & Horseshoe & DL & DP & BLasso & BElastic & Lasso & EN & Ridge \\
\hline \multicolumn{9}{|c|}{ ADNI } \\
\hline Accuracy & $0.842(0.736)$ & $0.831(0.747)$ & $0.842(0.747)$ & 0.907 & 0.852 & 0.907 & 0.907 & 0.902 \\
\hline Sensitivity & 0.810 & 0.796 & 0.810 & 0.964 & 0.810 & 0.964 & 0.964 & 0.993 \\
\hline Specificity & 0.935 & 0.935 & 0.935 & 0.739 & 0.978 & 0.739 & 0.739 & 0.630 \\
\hline AUC & $0.798(0.880)$ & $0.789(0.884)$ & $0.798(0.883)$ & 0.851 & 0.894 & 0.894 & 0.894 & 0.928 \\
\hline Brier Score & 0.107 & 0.109 & 0.110 & 0.093 & 0.113 & 0.093 & 0.093 & 0.098 \\
\hline \multicolumn{9}{|c|}{ OASIS } \\
\hline Accuracy & $0.733(0.741)$ & $0.733(0.688)$ & $0.733(0.741)$ & 0.827 & 0.800 & 0.827 & 0.840 & 0.787 \\
\hline Sensitivity & 0.818 & 0.818 & 0.818 & 1.000 & 0.977 & 1.000 & 1.000 & 1.000 \\
\hline Specificity & 0.613 & 0.613 & 0.613 & 0.581 & 0.548 & 0.581 & 0.613 & 0.484 \\
\hline AUC & $0.727(0.764)$ & $0.727(0.701)$ & $0.727(0.764)$ & 0.790 & 0.763 & 0.886 & 0.893 & 0.867 \\
\hline Brier Score & 0.129 & 0.130 & 0.129 & 0.173 & 0.128 & 0.173 & 0.160 & 0.213 \\
\hline \multicolumn{9}{|c|}{ Pima Indian Diabetes } \\
\hline Accuracy & 0.727 & 0.727 & 0.727 & 0.727 & 0.708 & 0.727 & 0.734 & 0.734 \\
\hline Sensitivity & 0.705 & 0.705 & 0.705 & 0.867 & 0.667 & 0.867 & 0.876 & 0.895 \\
\hline Specificity & 0.776 & 0.776 & 0.776 & 0.429 & 0.796 & 0.429 & 0.429 & 0.388 \\
\hline AUC & 0.711 & 0.711 & 0.711 & 0.648 & 0.731 & 0.682 & 0.692 & 0.696 \\
\hline Brier Score & 0.197 & 0.197 & 0.197 & 0.273 & 0.199 & 0.273 & 0.266 & 0.266 \\
\hline \multicolumn{9}{|c|}{ Colon } \\
\hline Accuracy & 0.846 & 0.769 & 0.769 & 0.923 & 0.769 & 0.923 & 0.923 & 0.923 \\
\hline Sensitivity & 1.000 & 0.750 & 0.750 & 1.000 & 1.000 & 1.000 & 1.000 & 1.000 \\
\hline Specificity & 0.778 & 0.778 & 0.778 & 0.889 & 0.667 & 0.889 & 0.889 & 0.889 \\
\hline AUC & 0.889 & 0.764 & 0.764 & 0.944 & 0.833 & 0.944 & 0.944 & 0.944 \\
\hline Brier Score & 0.121 & 0.224 & 0.240 & 0.077 & 0.276 & 0.077 & 0.077 & 0.077 \\
\hline
\end{tabular}


Figures
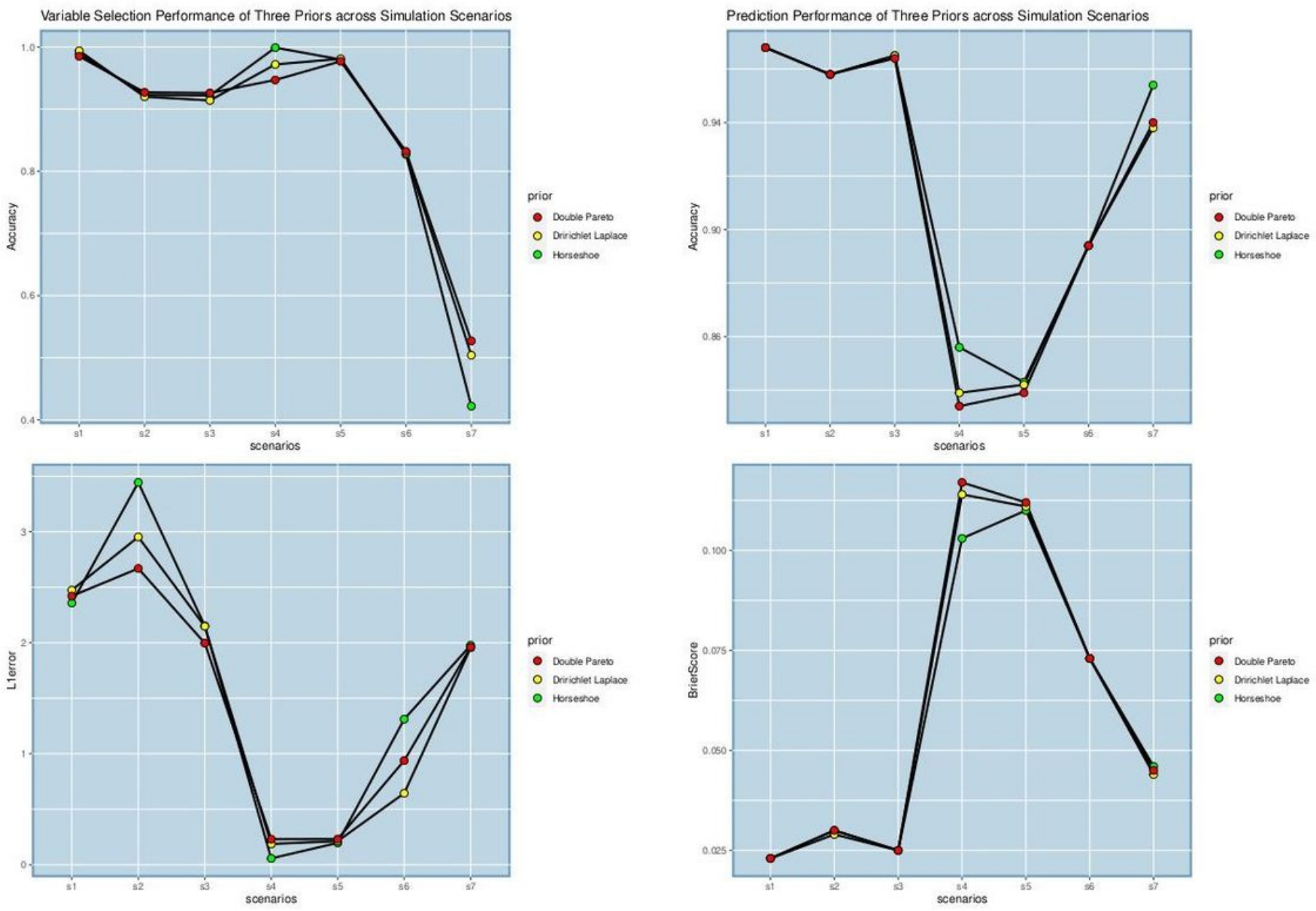

(a) Variable Selection Performance

(b) Prediction Performance

\section{Figure 1}

Prediction \& Variable Selection Performance in LR with Shrinkage Priors across Simulation Scenarios 


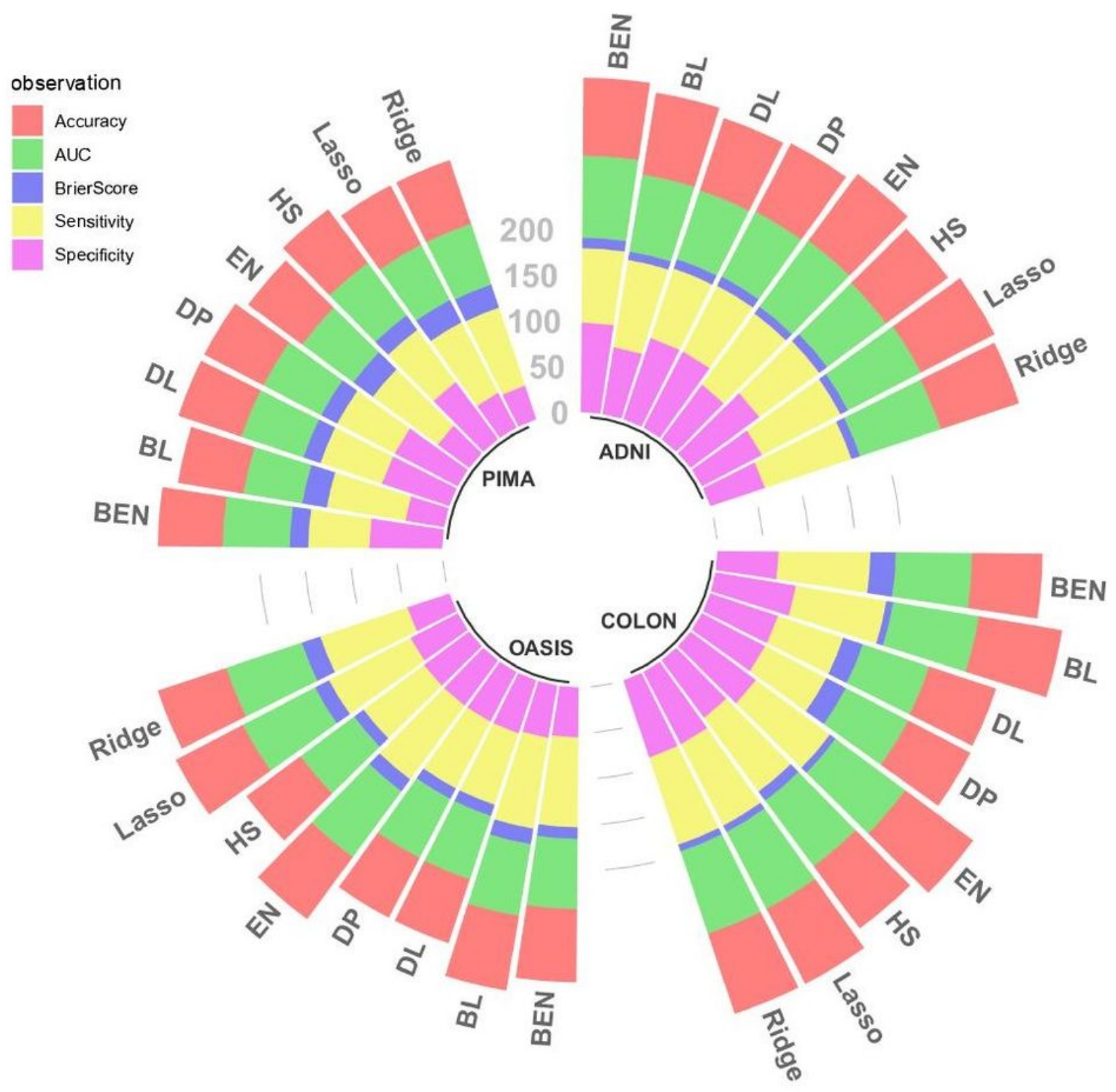

Figure 2

Circular Bar Chart comparing Prediction Metrics among datasets 


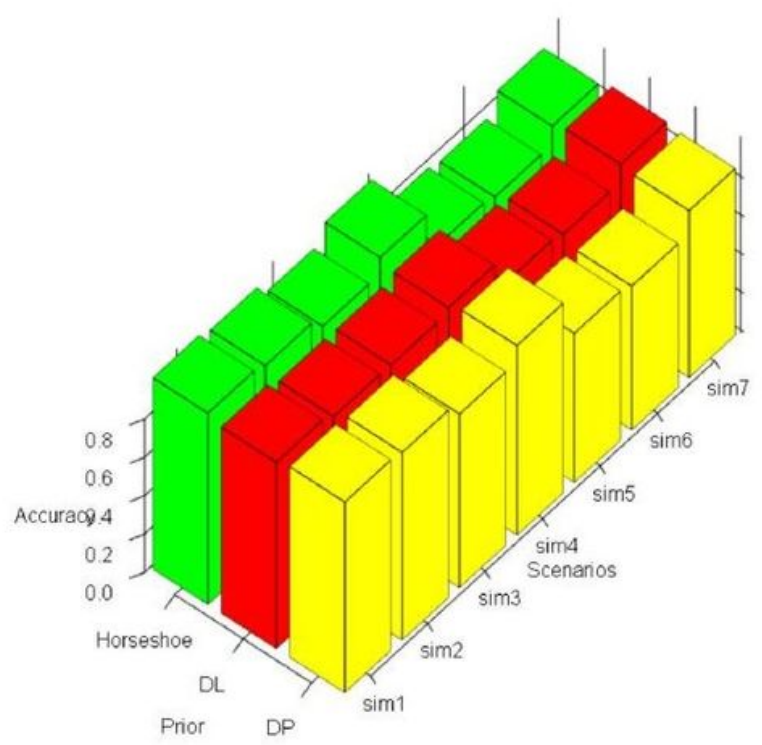

(a) Variable Selection Performance

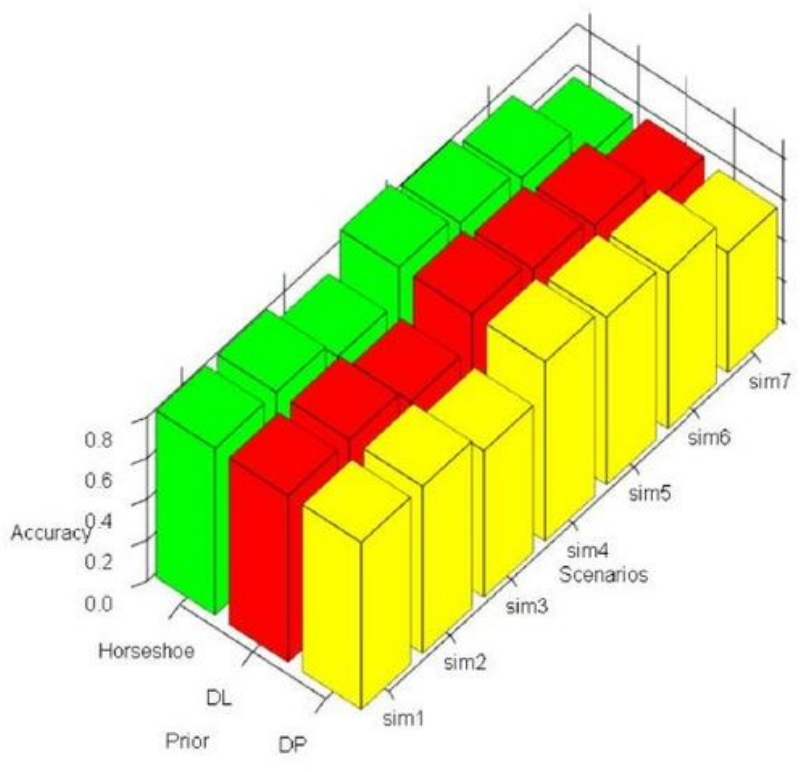

(b) Prediction Performance

Figure 3

Prediction \& Variable Selection Performance in MLR with Shrinkage Priors across Simulation Scenarios 


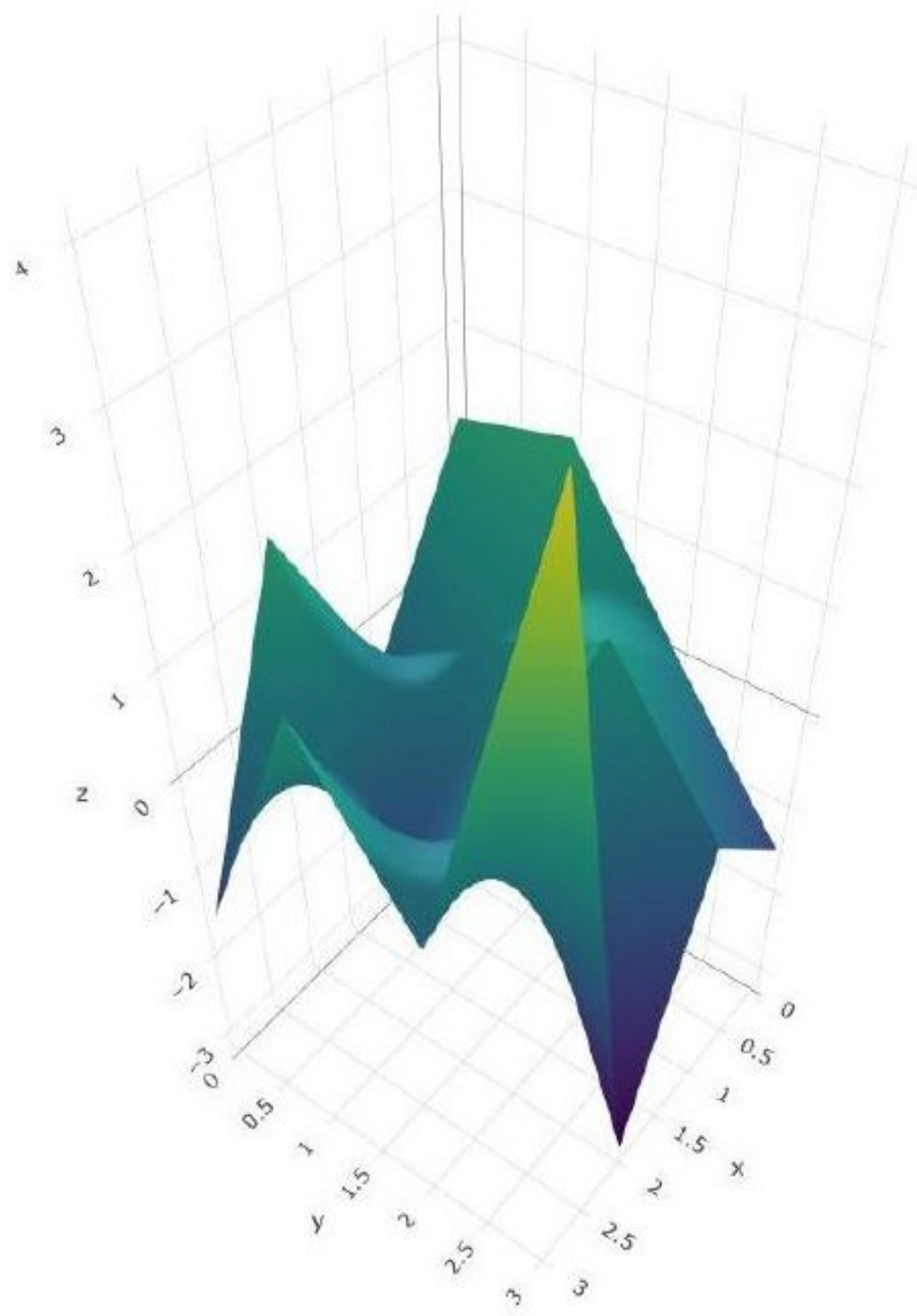

Figure 4

ROC Surface Plot for Horseshoe prior in data sets 